\title{
Rational design of stable sulfur vacancies in molybdenum disulfide for hydrogen
} evolution

Zhao, Yunxing; Tang, Michael T.; Wu, Sudong; Geng, Jing; Han, Zhaojun; Chan, Karen; Gao, Pingqi; Li, Hong

Published in:

Journal of Catalysis

Link to article, DOI:

10.1016/j.jcat.2019.12.028

Publication date:

2020

Document Version

Peer reviewed version

Link back to DTU Orbit

Citation (APA):

Zhao, Y., Tang, M. T., Wu, S., Geng, J., Han, Z., Chan, K., Gao, P., \& Li, H. (2020). Rational design of stable sulfur vacancies in molybdenum disulfide for hydrogen evolution. Journal of Catalysis, 382, 320-328.

https://doi.org/10.1016/j.jcat.2019.12.028

\section{General rights}

Copyright and moral rights for the publications made accessible in the public portal are retained by the authors and/or other copyright owners and it is a condition of accessing publications that users recognise and abide by the legal requirements associated with these rights.

- Users may download and print one copy of any publication from the public portal for the purpose of private study or research.

- You may not further distribute the material or use it for any profit-making activity or commercial gain

- You may freely distribute the URL identifying the publication in the public portal 


\section{Rational Design of Stable Sulfur Vacancies in Molybdenum Disulfide}

\section{for Hydrogen Evolution}

Yunxing Zhao ${ }^{\mathrm{a}, \mathrm{b}, \uparrow}$, Michael T. Tang ${ }^{\mathrm{c}, \uparrow}$, Sudong $\mathrm{Wu}^{\mathrm{b}, \uparrow}$, Jing Geng ${ }^{\mathrm{b}, \uparrow}$, Zhaojun Han ${ }^{\mathrm{d}, \mathrm{e}}$, Karen Chan, ${ }^{\mathrm{f}, *}$ Pingqi Gao, ${ }^{\mathrm{g}, *}$ and Hong $\mathrm{Li}^{\mathrm{a}}{ }^{\mathrm{a}, \mathrm{i}, *}$,

${ }^{a}$ School of Mechanical and Aerospace Engineering, Nanyang Technological University, 639798, Singapore

${ }^{b}$ Ningbo Institute of Material Technology and Engineering, Chinese Academy of Sciences, Ningbo 315201, China

${ }^{\mathrm{c}}$ SUNCAT Center for Interface Science and Catalysis, Department of Chemical Engineering, Stanford University, California 94305, USA

${ }^{\mathrm{d} C S I R O}$ Manufacturing, PO Box 218, 36 Bradfield Road, Lindfield, NSW 2070, Australia

eSchool of Chemical Engineering, UNSW Sydney, NSW 2052, Australia

${ }^{f}$ Catalysis Theory Center, Department of Physics, Technical University of Denmark, Lyngby, Denmark 2820

${ }^{g}$ School of Materials, Sun Yat-sen University, Guangzhou 510275, China

${ }^{\mathrm{h}}$ Centre for Micro-/Nano-electronics (NOVITAS), School of Electrical and Electronic Engineering, Nanyang Technological University, 639798, Singapore

${ }^{\mathrm{i}}$ CINTRA CNRS/NTU/THALES, UMI 3288, Research Techno Plaza, 637553, Singapore

${ }^{\dagger}$ equal contribution

*corresponding authors. Karen Chan: kchan@fysik.dtu.dk; Pingqi Gao: gaopq3@mail.sysu.edu.cn; Hong Li: ehongli@ntu.edu.sg 
ABSTRACT: Sulfur (S) vacancies in $\mathrm{MoS}_{2}$ have been found to act as a new active center, which shows an unprecedented intrinsic HER activity under elastic strain. However, such S-vacancies are unstable and the activities are very sensitive to the vacancy concentration. A strategy to stabilize these abundant active sites is thus highly desirable. Herein, we rationally design a catalyst system to stabilize $\mathrm{S}$-vacancies in the basal plane of $2 \mathrm{H}-\mathrm{MoS}_{2}$ supported on defective vertical graphene network (VGN). The energetically favorable line-shaped $\mathrm{S}$-vacancies in $\mathrm{MoS}_{2}$ shows a consistently high HER activity that is insensitive to S-vacancy concentration. Moreover, the defective graphene support effectively stabilizes these S-vacancies. The optimized catalyst exhibits a superior HER activity with overpotential of $128 \mathrm{mV}$ at $10 \mathrm{~mA} \mathrm{~cm}{ }^{-2}$ and Tafel slope of $50 \mathrm{mV} \mathrm{dec}{ }^{-1}$. Most importantly, the catalyst shows greatly increased stability over $500 \mathrm{~h}$; benchmarking the most stable nonprecious HER catalyst in acidic media to date.

KEYWORDS: 2D heterostructure, hydrogen evolution, stabilization function, vacancies, long-term stability 


\section{Introduction}

As a clean and sustainable energy resource, hydrogen has been considered to be one of the most promising candidates for replacing traditional fossil fuels in the future [1-3]. Water electrolysis for hydrogen generation through the hydrogen evolution reaction (HER) is a renewable and sustainable technology, yet it only contributes $4 \%$ of the global hydrogen production [4]. The majority of this $4 \%$ hydrogen is produced via alkaline water electrolysis (AWE) that can be implemented in large-scale owing to its excellent scalability and competitive cost [5-7]. The other commercial water electrolysis technology, proton exchange membrane (PEM) electrolysis, shows many advantages over AWE, including high-purity pressurized hydrogen, good partial load range (i.e., compatible with intermittent renewable energy sources), high efficiency, and portable/compact device [1]. However, it has poor scalability because its noble metal-based catalysts prevent it from large-scale implementation, and also increase the cost significantly $[1,8]$. Over five-year global platinum $(\mathrm{Pt})$ production is needed to produce enough hydrogen to meet our annual energy consumption with current PEM electrolysis technology.

Bio-inspired HER catalyst molybdenum disulfide $\left(\mathrm{MoS}_{2}\right)$, which is 10,000 times more abundant than Pt, has shown great potential as an alternative to Pt catalyst in PEM electrolyzer [8]. Three types of HER active sites in $\mathrm{MoS}_{2}$ have been discovered in the past decade, including edge-site [9,10], 1T-phase metallic clusters [11], and sulfur vacancies (S-vacancies) in $2 \mathrm{H}$-phase basal plane [12-18]. The edge sites show extraordinary activity, however, its low density necessitates ultrasmall nanoparticles uniformly distributed and stabilized on conductive substrate $[10,19] .1 T$-phase $\mathrm{MoS}_{2}$ is rich in active sites but it is metastable [11]. The recently discovered S-vacancy is abundant in the basal plane of $\mathrm{MoS}_{2}$, and shows unprecedented intrinsic activity under elastic strain [16, 20,21]. However, it is unstable particularly at high concentration; and its HER activity is very sensitive to S-vacancy concentration (\%S-vacancy) [13, 17]. Finding ways to stabilize these abundant S-vacancies active sites remains an open challenge. Moreover, the HER activity shows strong dependence on $\% \mathrm{~S}$-vacancy, resulting in performances variation in large-area catalysts where local S-vacancy distribution and concentration are difficult to control.

In this work, an optimal $\mathrm{MoS}_{2}$-based HER catalyst is rationally designed by using density functional theory (DFT) calculations, and then constructed using vertical defective graphene as the platform to stabilize S-vacancies in $\mathrm{MoS}_{2}$. The unique features of this design contain (1) the two-dimensional (2D) graphene with defects help to stabilize the 2D $\mathrm{MoS}_{2}$ 
with S-vacancies $\left(\mathrm{V}-\mathrm{MoS}_{2}\right)$ by forming a stable 2D-2D interface, (2) the 2D graphene facilitates the growth of thin $\mathrm{MoS}_{2}$ catalysts, (3) robust catalytic activity that is insensitive to \%S-vacancy by generating line-shaped S-vacancies, (4) standing vertical graphene network (VGN) directly grown on conductive carbon substrate minimizes Ohmic loss, and (5) open structure of VGN facilitates proton transport in HER process. The resulting V-MoS 2 /VGN catalyst shows excellent HER activity and extraordinary stability.

\section{Experimental Section}

2.1. Synthesis of $\mathrm{V}-\mathrm{MoS}_{2} / \mathrm{VGN}$ on Carbon Paper $\left(\mathrm{V}-\mathrm{MoS}_{2} / \mathrm{VGN} @ \mathrm{CP}\right), \mathrm{MoS}_{2} @ \mathrm{CP}$ and VGN@CP structures

Carbon paper (CP, TGP-H-060) was purchased from Toray Co. Ltd. Vertically standing graphene were prepared on the $\mathrm{CP}$ substrate by a rapid mesoplasma chemical vapor deposition (MPCVD) process at $900{ }^{\circ} \mathrm{C}(18 \mathrm{~kW}, 13.56 \mathrm{MHz})$. The as-prepared substrates were denoted as VGN@CP, i.e., VGN on carbon paper substrate. $\mathrm{MoS}_{2} / \mathrm{VGN} @ \mathrm{CP}$ sample were then synthesized via solvothermal method. $30 \mathrm{mg}$ of $\left(\mathrm{NH}_{4}\right)_{2} \mathrm{MoS}_{4}$ powder was firstly dispersed in $30 \mathrm{ml} \mathrm{N}$, N-dimethylformamide (DMF) by ultrasonication and continuously stirred for $30 \mathrm{~min}$ at room temperature. Afterward, a piece of VGN@CP (1 x $4 \mathrm{~cm})$ was immersed in the solution and transferred into a $50 \mathrm{ml}$ Teflon-lined stainless steel autoclave. The solvothermal process was carried out in an oven at $200{ }^{\circ} \mathrm{C}$ for $15 \mathrm{~h}$ followed by natural cooling down to room temperature. After washing with deionized water and alcohol, the final products were obtained by drying under vacuum at $70{ }^{\circ} \mathrm{C}$ for $10 \mathrm{~h} . \mathrm{MoS}_{2}$ directly grown on CP was also prepared with the same solvothermal method to obtain $\mathrm{MoS}_{2} @ \mathrm{CP}$ structure as a control sample.

The mild argon (Ar) plasma was generated by dispersing a $30 \mathrm{~W}$ RF power in a 6-inch diameter vacuum chamber at a pressure of 0.1 mbar. The as-prepared $\mathrm{MoS}_{2} / \mathrm{VGN} @ \mathrm{CP}$ sample were exposed at the chamber with different times $(20,40,60,80,100,120,140$ and $160 \mathrm{~s}$, respectively) to obtain the V-MoS $/$ VGN@CP samples.

\subsection{Electrochemical Measurements}

Electrochemical measurements were carried out by an electrochemical workstation (CHI Instrument 660E) with a standard three-electrode setup in a $0.5 \mathrm{M} \mathrm{H}_{2} \mathrm{SO}_{4}$ solution. V-MoS $2 / \mathrm{VGN} @ \mathrm{CP}, \mathrm{MoS}_{2} / \mathrm{VGN} @ \mathrm{CP}, \mathrm{MoS}_{2} @ \mathrm{CP}, \mathrm{VGN} @ \mathrm{CP}$ and CP samples were used as working electrodes. $\mathrm{Ag} / \mathrm{AgCl}$ (saturated $\mathrm{KCl}$ ) electrode and $\mathrm{Pt}$ foil were used as reference 
electrode and counter electrode, respectively. All potentials were converted to a reversible hydrogen electrode (RHE) scale, i.e., $\mathrm{RHE}=\mathrm{V}$ vs. $\mathrm{Ag} / \mathrm{AgCl}+0.197+0.059 \times \mathrm{pH}$. The catalytic performance was measured by linear sweeping from -1 to $0 \mathrm{~V}$ (vs. RHE) with a scan rate of $5 \mathrm{mV} \mathrm{s}^{-1}$, with $85 \%$ iR correction compensation for all LSV curves from electrochemical workstation, where the Ohmic series resistance Rs is obtained under open-circuit voltage. Faradic efficiency was tested on Agilent 7890A GC equipment under flow cell with Ar as carrier gas, and the interval time is $1311 \mathrm{~s}$.

\subsection{DFT Calculation Details}

DFT calculations were performed using the Atomic Simulation Envinement (ASE) with Quantum Espresso code using plane-wave ultrasoft pseudopotentials.[22, 23] The BEEF-vdW functional was chosen as it provides a reasonable description of van der Waals forces between $\mathrm{MoS}_{2}$ and graphene layers while maintaining an accurate prediction of chemisorption energies.[24] We noted that the functional was tested with empirical data, and its error on adsorption energies was found to be within 0.1-0.2eV.[24] To ensure no lattice strain effects were present when adsorbing a single $\mathrm{MoS}_{2}$ layer on to graphene, we modelled our heterostructure system by matching the lattice parameters of graphene in a $(9 \times 3)$ rectangular lattice cell containing 108 carbon atoms closely with $\mathrm{MoS}_{2}$ in a (4x4) rectangular lattice cell containing $16 \mathrm{Mo}$ atoms and $32 \mathrm{~S}$ atoms. A PWSCF input script that recreates the heterostructure in its supercell is provided in the Supplementary Information. To reduce computing time, plane-wave and density cutoffs were 400 and $4000 \mathrm{eV}$, respectively, with a Fermi-level smearing width of $0.1 \mathrm{eV}(0.0073 \mathrm{Ry})$ and $2 \times 2 \times 1 k$-point sampling; convergence with respect to plane-wave cutoffs and $k$ points were verified in a previous report.[25, 26] Still, a convergence study is provided in Supplementary Table S1, where we showed that $\mathrm{H}^{*}$ and heterostructure adsorption trends, to the extent of the pristine and V-MoS 2 with 2 S-vacancies over pristine graphene system, remain unchanged through energy cutoffs of 550 and $5500 \mathrm{eV}$, and $3 \times 3 \times 1 \mathrm{k}$-point sampling. Periodic boundary conditions were applied and 11 $\AA ̊$ of vacuum was used in between the periodic heterostructure layers. Calculations performed were spin-restricted as the model systems are non-magnetic. All structures were fully relaxed until all force components were less than $0.05 \mathrm{eV} \AA^{-1}$; no atoms were frozen in the supercell. We define adsorption energy of hydrogen for our system to be:

$$
\Delta \mathrm{E}\left(\mathrm{H}^{*}\right)=\mathrm{E}\left(\mathrm{MoS}_{2}+\mathrm{H}+\text { graphene }\right)-\mathrm{E}\left(\mathrm{MoS}_{2}+\text { graphene }\right)-1 / 2 * \mathrm{E}\left(\mathrm{H}_{2}\right)
$$

where $\mathrm{E}\left(\mathrm{MoS}_{2}+\mathrm{H}+\right.$ graphene $)$ is the total energy for a given $\mathrm{MoS}_{2}$ system with a given graphene support and $\mathrm{n}$ hydrogen atoms adsorbed on basal plane, $\mathrm{E}\left(\mathrm{MoS}_{2}+\right.$ graphene $)$ is the 
total energy of the clean system, and $\mathrm{E}\left(\mathrm{H}_{2}\right)$ is the energy of a gas phase hydrogen molecule. The Gibbs free energy for hydrogen adsorption was then calculated as

$$
\Delta \mathrm{G}\left(\mathrm{H}^{*}\right)=\Delta \mathrm{E}\left(\mathrm{H}^{*}\right)+\Delta \mathrm{E}_{\mathrm{ZPE}}-\mathrm{T}^{*} \Delta \mathrm{S}_{\mathrm{H}}
$$

where $\Delta \mathrm{E}_{\mathrm{ZPE}}$ is the zero-point energy difference between the adsorbed state and the gas phase state of the system, and $\Delta \mathrm{S}_{\mathrm{H}}$ is the entropy difference between the adsorbed state and the gas phase standard state ( $300 \mathrm{~K}, 1 \mathrm{bar}$ ) of the system. Here we have approximated the entropy of hydrogen adsorption as $\Delta \mathrm{S}_{\mathrm{H}} \approx 1 / 2\left(\mathrm{~S}_{\mathrm{H}_{2}}{ }^{\circ}\right)$, where $\mathrm{S}_{\mathrm{H}_{2}}{ }^{\circ}$ is the entropy of gas phase $\mathrm{H}_{2}$ at standard condition. The adsorption energy of $\mathrm{MoS}_{2}$ on the graphene support was defined by:

$$
\Delta \mathrm{E}(\text { ads })=\left(\mathrm{E}\left(\mathrm{MoS}_{2}+\text { graphene }\right)-\mathrm{E}\left(\mathrm{MoS}_{2}\right)+\mathrm{E}(\text { graphene })\right) / \mathrm{N}_{\mathrm{C}}
$$

where the adhesion energy of $\mathrm{MoS}_{2}$ on the support was normalized to the number of carbon atoms $\mathrm{N}_{\mathrm{C}}$ in the supercell.

\section{Results and Discussion}

\subsection{Rational Design of Catalyst}

The use of support interactions to stabilize a catalytic material has been explored recently [25, 27-30]. We rationalized an optimal design for the following catalyst system (see Fig. 1 for schematic) using DFT calculations. Conductive 2D graphene is chosen as the catalyst support to form a stable 2D-2D catalyst-support interface with $2 \mathrm{D} \mathrm{MoS}_{2}$ catalyst. Pristine and defective graphenes would have different interactions with $\mathrm{MoS}_{2}$ due to their distinct chemical reactivity. We thus firstly study the adhesion energy between $\mathrm{MoS}_{2}$ and various graphenes. Our model system consists of a single infinite layer of $\mathrm{MoS}_{2}$ with $4 \mathrm{Mo}$ atoms in the $x$ direction and 8 atoms in the $y$ direction (pristine $\mathrm{MoS}_{2}$ ), stacked on a single infinite layer of graphene composed of 108 carbon atoms (pristine graphene), and defects were created on $\mathrm{MoS}_{2}$ or graphene based on the perfect stacked bilayer. We started by elucidating the most stable arrangement of S-vacancies on graphene support before investigating the $\mathrm{H}$ adsorption strength.

The S-vacancies that are observed most frequently in experiment are point and line-shaped defects $[31,32]$. We thus compare the energetics of line-shaped defects on the basal plane of $\mathrm{MoS}_{2}$ to that of randomly generated point defects. We assume that a uniform arrangement of individual S-vacancies across the top layer of sulfur atoms on the $\mathrm{MoS}_{2}$ sheet will yield randomly distributed point defects because those individual S-vacancies are well separated and have no influence on each other. For the sake of computational time, we 
limited this stability study on defective graphene to those with even numbers of $\mathrm{C}$-vacancies. However, our simulations showed that the most stable configuration of S-vacancies in $\mathrm{MoS}_{2}$ anchored on defective graphene is line-shaped, in agreement with previous report [33] (see Supplementary Figure S1 for more details).

This line-shaped configuration results in hydrogen adsorption free energies that are insensitive to the $\% \mathrm{~S}$-vacancy, as depicted in Fig. 2. We explored in depth the changes to the free energy diagram as we introduce line-shaped S-vacancies in $\mathrm{MoS}_{2}$ supported on various graphene systems, as depicted in the insets of Fig. 2a (pristine graphene, i.e., $0 \%$ $\mathrm{C}$-vacancies), 2b (2 carbon atoms missing, i.e., 1.85\% C-vacancies), $2 \mathrm{c}$ (4 carbon atoms missing, i.e., 3.70\% C-vacancies), $2 \mathrm{~d}$ (an aromatic ring of 6 carbon atoms missing, i.e., $5.56 \%$ $\mathrm{C}$-vacancies), 2e (2 aromatic rings of 12 carbon atoms missing, i.e., 11.11\% C-vacancies), and $2 \mathrm{f}$ (graphene edge, i.e., 50\% C-vacancies). The atomic configuration of the heterostructure with graphene on top is presented in order to show clearly the C-vacancies in graphene (the blue dots in the insets). For each free energy diagram, we studied how $\mathrm{H}$ adsorption on the $\mathrm{MoS}_{2}$ basal plane is affected by the amount of line-shaped S-vacancies, i.e., $0.00 \%$ (blue), $3.13 \%$ (green), 6.25\% (orange), 9.38\% (cyan), and 12.50\% (red), as shown in Fig. 2 (see more defects configuration in Supplementary Figure S2). We limited our study to $12.50 \% \mathrm{~S}$-vacancy because $\mathrm{MoS}_{2}$ was found to become very unstable with S-vacancy above $12 \%$ [16]. The topmost blue curve shows the energetics on the pristine $\mathrm{MoS}_{2}$ over the 6 defective graphene systems. Without any S-vacancies, the $\mathrm{H}$ adsorption onto $\mathrm{MoS}_{2}$ basal plane is found to be very endergonic across all graphene systems, in agreement with previous studies that show the pristine $\mathrm{MoS}_{2}$ basal plane to be chemically inert $[9,10]$. The $\mathrm{H}$ adsorption energy drastically decreases when 3.13\% line-shaped S-vacancies are introduced. Following that, it barely changes when S-vacancy concentration increases from $3.13 \%$ to $12.50 \%$, and it remains around $-0.10 \mathrm{eV}$ (see Table S2 for detailed values), which indicates excellent HER activity.

We note that when $\mathrm{MoS}_{2}$ is adhered onto pristine graphene (Fig. 2a), and the line-shaped $\mathrm{S}$-vacancies are found to exhibit positive $\mathrm{H}$ binding energies at all \% $\mathrm{S}$-vacancy. When there are defects on the graphene systems, the $\mathrm{H}$ binding energy on $\mathrm{V}-\mathrm{MoS}_{2}$ drastically decreases towards negative values. The most optimal $\mathrm{H}$ binding across all \% S-vacancy occur when there are 4-6 C-vacancies on the graphene. When the graphene has less than 4 or more than 6 $\mathrm{C}$-vacancies, a weakening of the $\mathrm{H}$ binding energy is observed for $\mathrm{V}-\mathrm{MoS}_{2}$. Nevertheless, all line-shaped S-vacancies shows a small $\mathrm{H}$ absorption energy $(<0.16 \mathrm{eV})$ on all defective graphene systems. 
We also examined point-shaped S-vacancies in comparison to line-shaped ones. Over pristine graphene, we found similar $\mathrm{H}$ adsorption trends to that in literature [16] (see Supplementary Figure S3), where increasing \%S-vacancy leads to increasing $\mathrm{H}$ binding energies. Over defective graphene with $6 \mathrm{C}$-vacancies, we found that the $\mathrm{H}$ adsorption trend remains similar to those over the pristine graphene. This is in obvious contrast to line-shaped S-vacancies, where pristine graphene and defective graphene supports made significant difference on the trend of $\mathrm{H}$ adsorption upon increasing \% $\%$-vacancy. We speculate that the difference in $\mathrm{H}$ adsorption behavior between line-shaped S-vacancies and point-shaped S-vacancies on $\mathrm{MoS}_{2}$ over defective graphene systems may stem from the size of the S-vacancy site. Nevertheless, we note that for both line-shaped and point-shaped S-vacancies, the most stable $\% \mathrm{~S}$-vacancy hovers around $6.25 \%$ and $12.5 \%$. Putting together, our $\mathrm{H}$ free energy calculations suggest that the HER activity is mostly insensitive to the $\% \mathrm{~S}$-vacancy in the range of $6.25 \%$ to $12.5 \%$ when $\mathrm{V}-\mathrm{MoS}_{2}$ is supported on defective graphene - this is unique for line-shaped S-vacancies instead of point-shaped S-vacancies.

The stabilization of $\mathrm{V}-\mathrm{MoS}_{2}$ can be rationalized through the adhesion energetics between the V-MoS 2 layer and the defective graphene layer. Graphene interacts with the V-MoS layer via its $\pi$-conjugation layer. Carbon defects introduce defect states near the Fermi level in the graphene layer [34]. Similarly, when S-vacancies are introduced, gap states form near the Fermi level of adhered V-MoS 2 layer, which may be regarded as the active basal site for HER [16]. We hypothesize that, analogous to how $\mathrm{H}$ will stabilize S-vacancies on the $\mathrm{MoS}_{2}$ basal plane via chemisorption, the defect states on graphene stabilize the S-vacancies of $\mathrm{MoS}_{2}$ via physisorption, i.e., long-range Van-der Waals interactions. Adhesion between all $\mathrm{MoS}_{2}$ /graphene systems are exergonic; however, due to changes in adhesion area between $\mathrm{MoS}_{2}$ and defected graphene systems studied, we performed area normalization on the adhesion energy through the number of carbon atoms (108) in the supercell. Fig. 3a shows the adhesion energy between pristine $\mathrm{MoS}_{2}$ and defective graphene systems. Pristine $\mathrm{MoS}_{2}$ over pristine graphene is found to be the weakest adhered system of all the systems studied, consistent with previous report [25]. When a few C-vacancies are created in graphene, the adhesion energy becomes stronger. Specifically, the adhesion energy increases by $8.15 \%$ and $11.89 \%$ for defective graphene with a missing aromatic ring and graphene edge, respectively, in comparison with the pristine graphene. These observations suggest that defective graphene effectively stabilizes the supported V-MoS 2 above.

The influence of S-vacancies on the structure stability is presented in Fig. 3b, where the adhesion energy between $\mathrm{V}-\mathrm{MoS}_{2}$ and pristine graphene is presented. The introduction of the 
S-vacancies also drastically improves the adhesion energy between catalyst and support. Specifically, the adhesion energy increases by $6.30 \%$ and $12.49 \%$ for $\mathrm{MoS}_{2}$ with $3.03 \%$ and $12.50 \% \mathrm{~S}$-vacancies, respectively. The impacts of both S-vacancies in $\mathrm{MoS}_{2}$ and C-vacancies in graphene are summarized in the colored contour plot of adhesion energy, as shown in Fig. 3c. Both S- and C-vacancies increase the adhesion energy between catalyst and support, regardless of how the S-vacancies are arranged. We do note that the structure and phase of $\mathrm{MoS}_{2}$ may change at high \%S-vacancy; [16] and the conductivity of graphene will decrease at high $\% \mathrm{C}$-vacancy. Thus, one is not able to increase the stability continuously by simply increase the vacancy concentration.

\subsection{Experimental Fabrication and Evaluation of Catalyst}

As suggested by DFT calculations, conductive graphene with defects is crucial to make stable and efficient HER catalysts of $\mathrm{V}-\mathrm{MoS}_{2}$. However, one should balance the tradeoff between defects and conductivity in defective graphene. We thus choose a MPCVD system to directly grow VGN@CP as the conductive support. The high-energy $(18 \mathrm{~kW})$ plasma involved in the process results in rich defects in VGN while the high-temperature $\left(900{ }^{\circ} \mathrm{C}\right)$ ensures its excellent electrical conductivity, meanwhile thin layers range from 4 to 10 layers were obtained, as shown in Figure S4. Then $\mathrm{MoS}_{2}$ was grown on VGN@CP with a solvothermal method to form $\mathrm{MoS}_{2} / \mathrm{VGN} @ \mathrm{CP}$ structure [35]. We noted that the graphene substrate facilitated the growth of thin $\mathrm{MoS}_{2}$ flakes, instead of $\mathrm{MoS}_{2}$ particles/clusters as those on CP substrate (Supplementary Figure S5). Both interfaces, i.e., $\mathrm{MoS}_{2}-\mathrm{VGN}$ and VGN-CP, were formed by direct growth at elevated temperatures; leading to superior electrical contacts. Afterward, Ar plasma exposure was employed to create S-vacancies in $\mathrm{MoS}_{2}$, resulting in the final catalyst V-MoS $/ \mathrm{VGN} @ \mathrm{CP}$, as shown in Supplementary Figure S6. The open structure $\mathrm{MoS}_{2} / \mathrm{VGN} @ \mathrm{CP}$ support allows penetration of Ar plasma into the catalyst efficiently to create S-vacancies. The scanning electron microscopy (SEM) image of VGN reveals a three-dimensional (3D) porous network ( $\sim 200 \mathrm{~nm}$ in pore size and 2 3 $\mu \mathrm{m}$ in height), as shown in Fig. 4a. The large water contact angle of $147^{\circ}$ indicates a super hydrophobic surface of the as-prepared VGN (inset of Fig. 4a). The SEM image after the growth of $\mathrm{MoS}_{2}$ shows a well-retained VGN structure with a more closely packed surface, as depicted in Fig. 4b. The entire surface of VGN is covered with $\mathrm{MoS}_{2}$ thin sheets that have a much smaller size. The $\mathrm{MoS}_{2} / \mathrm{VGN} @ \mathrm{CP}$ surface remains hydrophobic with a slightly smaller water contact angle ( $131^{\circ}$ as depicted in the inset of Fig. $\left.4 b\right)$. 
In contrast, the catalyst surface became very hydrophilic after Ar plasma exposure, due to oxygen occupation (from water molecule) of S vacancy sites [36]. After 120-s Ar plasma exposure, the contact angle decreased to $25^{\circ}$, as displayed in the inset of Fig. 4c. The wettability of catalysts determines the infiltration ability and the mass transport of electrolyte, and thus largely influences the catalytic performance; [37] and the excellent wettability of our catalyst is favorable for high HER activity. It is worth noting that the catalyst surface became much rougher, yet the open network structure remained unchanged. The Raman spectra before and after Ar plasma exposure are similar (Fig. 4d), suggesting the surface chemical composition was not significantly changed. The $\mathrm{MoS}_{2} / \mathrm{VGN} @ \mathrm{CP}$ with a 120-s plasma treatment has exhibited the best performance that will be detailed later, we therefore use 120-s Ar plasma treatment as the optimum condition (i.e., $\mathrm{V}_{120}-\mathrm{MoS}_{2} / \mathrm{VGN} @ \mathrm{CP}$, where the subscript denotes 120-s Ar plasma exposure). To further examine the structure of the catalyst, particularly the $\mathrm{MoS}_{2} / \mathrm{VGN}$ interface, we performed high-resolution transmission electron microscopy (HRTEM). The TEM image of $\mathrm{MoS}_{2} / \mathrm{VGN}$ (Fig. 4e) shows that (1) both $\mathrm{MoS}_{2}$ and VGN sheets are thin enough to appear to be transparent under TEM, and (2) $\mathrm{MoS}_{2}$ (range of 2-7 layers) and graphene flakes form a uniform composite. A closer look reveals typical interplanar spacing of graphene and $\mathrm{MoS}_{2}$ to be about 3 and $6 \AA$, respectively, corresponding to the (002) planes of graphene and $2 \mathrm{H}-\mathrm{MoS}_{2}$ [35]. Moreover, thin $\mathrm{MoS}_{2}$ flakes are anchored on the surface of multilayer graphene flakes to form 2D heterostructure, suggesting VGN considerably limits the growth of thick $\mathrm{MoS}_{2}$ and its aggregation, which is beneficial for achieving high density of active sites. The plasma exposure did not change either the 3D open structure macroscopically or the lattice parameters microscopically, as displayed in Fig. 4f. It is worth noting that most $\mathrm{MoS}_{2}$ thin sheets align along with graphene sheets and form a stable 2D-2D interface, offering excellent contact that facilitates the electron transport because the electron conductivity across $\mathrm{MoS}_{2}$ layers is much poorer than that of in-plane transport [38, 39].

The electrocatalytic HER performances were evaluated in a typical three-electrode setup by steady-state linear sweep voltammetry (LSV) curves, as shown in Fig. 5a. $\mathrm{MoS}_{2} / \mathrm{VGN} @ \mathrm{CP}$ needs a much smaller potential of -217 mV than VGN@CP $(<-400 \mathrm{mV})$ and $\mathrm{MoS}_{2} @ \mathrm{CP}(-286 \mathrm{mV})$ to deliver a current density of $10 \mathrm{~mA} \mathrm{~cm}{ }^{-2}$, proving the importance of $\mathrm{MoS}_{2}$-graphene interface. Notably, $\mathrm{V}_{120}-\mathrm{MoS}_{2} / \mathrm{VGN} @ \mathrm{CP}$ only needs -86 mV to start hydrogen evolution and $-128 \mathrm{mV}$ to achieve $10 \mathrm{~mA} \mathrm{~cm}{ }^{-2}$; and the LSV curves show negligible change in argon environment or when carbon electrode was chosen as the counter electrode, as displayed in Figure S7a. The corresponding Tafel slope of 
$\mathrm{V}_{120}-\mathrm{MoS}_{2} / \mathrm{VGN} @ \mathrm{CP}$ is as low as $50 \mathrm{mV} \mathrm{dec}{ }^{-1}$ (Fig. 5b), much lower those that of the other samples, which indicates a much faster HER kinetic [40]. And the $\mathrm{H}_{2}$ Faradic efficiency of $\mathrm{V}_{120}-\mathrm{MoS}_{2} / \mathrm{VGN} @ \mathrm{CP}$ is close to $100 \%$, as depicted in Figure S7b. It is worth noting that $\mathrm{V}_{120}-\mathrm{MoS}_{2} / \mathrm{VGN} @ \mathrm{CP}$ also shows a much lower overpotential than those of similar catalyst systems, including $\mathrm{MoS}_{2}-\mathrm{VGN} @$ nickel foam ( 220 mV) [35], edge sites of $\mathrm{MoS}_{2}$ nanoparticles on reduced graphene oxide $(\sim 150 \mathrm{mV})$ [19], amorphous $\mathrm{MoS}_{\mathrm{x}}$ on graphene/carbon nanotube@CP (141 mV) [41], and 1T-MoS $2 /$ carbon cloth $(\sim 151 \mathrm{mV})$ [42]. Indeed, the HER performances of $\mathrm{V}_{120}-\mathrm{MoS}_{2} / \mathrm{VGN} @ \mathrm{CP}$ are comparable or better than the state-of-the-art $\mathrm{MoS}_{2}$-based catalysts (as summarized in Supplementary Table S3). Most importantly, the catalyst is very stable within 500-h continuous operation (Fig. 5c), which is 4 orders of magnitude more stable than monolayer S-vacancies supported on gold substrate [16]. This superior stability also benchmarks the nonprecious HER catalyst in acidic media (Supplementary Table S4). The 3D open structure of the catalyst morphology is well retained after 500-h stability test and no Pt peaks appeared in SEM-EDS element mapping, as shown in the SEM image (see inset of Fig. 5c, and Figure S8), due to the much low overpotential than those reported in the literatures $[43,44]$.

Next, we discuss how to determine the optimum Ar plasma exposure duration. We measured the dependence of overpotential (at $10 \mathrm{~mA} \mathrm{~cm}{ }^{-2}$ ) on plasma treatment time, as depicted in Fig. 5d (see Figure S7c and S7d for full LSV curves and Tafel slopes of samples with different Ar plasma exposure). One can see that the overpotential declines dramatically with the increment of plasma treatment time in the first $60 \mathrm{~s}$, and then gradually decreases to the lowest value of $128 \mathrm{mV}$ at $120 \mathrm{~s}$. Beyond $120 \mathrm{~s}$, the overpotential starts to increase, which is attributed to the structural damage of $\mathrm{MoS}_{2}$ since too many S-vancancies have been created [16]. The quick decrease of overpotential in the first $60 \mathrm{~s}$ is attributed to the increased hydrophilicity of the catalyst and the increment of HER active site density, so the activity is sensitive to $\% \mathrm{~S}$-vacancy below $60 \mathrm{~s}$. Interestingly, the stable performance between $60 \mathrm{~s}$ and $160 \mathrm{~s}$ suggest the robust HER performance that is insensitive to \%S-vacancy (Fig.5d and Figure S7c), consistent with our theoretic prediction (Fig. 2 and Supplementary Figure S3).

The energy dispersive X-ray spectroscopy (EDS) analysis (Supplementary Figure S9) shows that the atomic ratio of $\mathrm{S}: \mathrm{Mo}$ is $\sim 2.1$, consistent with $\mathrm{MoS}_{2}$ made using solvothermal method [45]. EDS mapping images reveal the uniform distribution of $\mathrm{C}$, Mo, and S elements in the $\mathrm{MoS}_{2} / \mathrm{VGN} @ \mathrm{CP}$ sample. Electron paramagnetic resonance (EPR) was used to confirm the evolution of the defects concentration, as depicted in Fig. 5e. Increasing Ar plasma exposure time from 0 to $160 \mathrm{~s}$ led to enhanced EPR intensity $(\mathrm{g}=2.006)$, indicating 
consistently increased \% $\%$-vacancy [46, 47]. The S-vacancy concentration is further quantified by X-ray photoelectron spectroscopy (XPS). The evolution of XPS spectra of $\mathrm{V}_{\mathrm{t}}-\mathrm{MoS}_{2} / \mathrm{VGN}$, where $\mathrm{t}$ is the Ar plasma exposure time, was measured when $\mathrm{t}$ increases from 0 (i.e., $\mathrm{MoS}_{2} / \mathrm{VGN} @ \mathrm{CP}$ ) to $160 \mathrm{~s}$. One can see that $\mathrm{S} 2 \mathrm{p}$ state is significantly weakened by plasma exposure due to formation of S-vacancies on the catalyst surface (see Fig. 5f). Also, the Mo 3d state becomes weaker after plasma exposure (see Supplementary Figure S10). All Mo peaks including $\mathrm{Mo}^{4+}(232.3 \mathrm{eV} / 229.0 \mathrm{eV})$ and its slightly oxidized $\mathrm{Mo}^{5+}$ (233.6 $\mathrm{eV} / 230.3 \mathrm{eV})$ and $\mathrm{Mo}^{6+}(235.8 \mathrm{eV})[48,49]$ were all taken into account for quantification, and the atomic ratio of S:Mo is calculated to be $\sim 2.11$ for $\mathrm{t}=0$ curve based on the XPS spectra, consistent with the aforementioned EDS measurement. As Ar plasma treatment time increases, the ratios of S: Mo decrease gradually, indicating more S-vacancy was created (see Table S5 for atomic ratios and S-vacancy concentration). For the optimum condition $\mathrm{t}=120 \mathrm{~s}$ (i.e., $\mathrm{V}_{120}-\mathrm{MoS}_{2} / \mathrm{VGN} @ \mathrm{CP}$ highlighted in red), S:Mo atomic ratio is decreased to $\sim 1.86$, corresponding to $7 \%$ (normalized stoichiometric ratio 2.0 ) or $12 \%$ S-vacancy (normalized to 2.11 as measured).

To estimate the accessible active sites of the electrodes, the electrochemical capacitance $\mathrm{C}_{\mathrm{dl}}$ is calculated in the double layer capacitance region, as shown in Supplementary Figure S11a (see Supplementary Figure S12 for the corresponding CV scans). The pristine $\mathrm{MoS}_{2} / \mathrm{VGN} @ \mathrm{CP}$ shows an $\mathrm{C}_{\mathrm{dl}}$ of $10.6 \mathrm{mF} \mathrm{\textrm {cm } ^ { - 2 }}$, significantly higher than that of $\mathrm{MoS}_{2} @ \mathrm{CP}$ $\left(4.6 \mathrm{mF} \mathrm{cm}{ }^{-2}\right)$, which arises from the much thinner and better-separated $\mathrm{MoS}_{2}$ thin sheets on VGN than those on CP. More importantly, $\mathrm{V}_{120}-\mathrm{MoS}_{2} / \mathrm{VGN} @ \mathrm{CP}$ displays a large $\mathrm{C}_{\mathrm{dl}}$ of 13.6 $\mathrm{mF} \mathrm{cm}{ }^{-2}$, indicating that $\mathrm{S}$-vacancies effectively increase the active surface area in the basal plane. For $\mathrm{V}_{\mathrm{t}}-\mathrm{MoS}_{2} / \mathrm{VGN} @ \mathrm{CP}$ with different Ar plasma treatment time, we found the $\mathrm{C}_{\mathrm{dl}}$ and electrochemical active surface area (ECSA, calculated from measured $\mathrm{C}_{\mathrm{dl}}$ with reference to the capacitance $\left(60 \mu \mathrm{F} / \mathrm{cm}^{2}\right)$ of the flat $\mathrm{MoS}_{2}$ [50]) increases generally (Figure $\mathrm{S} 11 \mathrm{~b}$ and $\mathrm{S} 11 \mathrm{c})$, reveling the surface roughness increases from $\mathrm{t}=0 \mathrm{~s}$ to $160 \mathrm{~s}$. And the ECSA-corrected LSV curves (Figure S11d) also reflect the same trend that activity change slightly from $60 \mathrm{~s}$ to $160 \mathrm{~s}$, consistent with DFT prediction. It is worth noting that the Ohmic loss is negligible, owing to the superior conductivity of defective graphene support.

For the potential application of our catalyst in industry, one of the advantages is the low cost of $\mathrm{MoS}_{2}$ (more than 10,000-time more abundant and 1000-time cheaper than $\mathrm{Pt}$ ), and the superior activity and long-term stability also make it promising for practical use; thus it has 
great potential for large-scale implementation. Nevertheless, two major challenges remain. Firstly, our V-MoS 2 /VGN catalyst was directly grown on carbon support, and the process is not compatible with industrial PEM powder catalysts (Pt particle powder loaded on carbon support). A method to transfer the V-MoS $2 @ V G N$ catalyst onto proton-exchange membrane has to be developed. Besides, the reported methods for S-vacancy generation in $\mathrm{MoS}_{2}$, including plasma exposure and electrochemical reduction, are not suitable for treatment of $\mathrm{V}-\mathrm{MoS}_{2} / \mathrm{VGN}$ powder, so a scalable process to generate $\mathrm{S}$-vacancies in $\mathrm{MoS}_{2}$ powder is needed before a practical application can be realized.

\section{Conclusion}

In summary, we have designed and evaluated a strategy to stabilize S-vacancies for HER catalyst. $\mathrm{MoS}_{2}$ thin sheets with $\mathrm{S}$-vacancies are anchored on defective and conductive graphene that forms an open network, which allows generation of S-vacancies using $\mathrm{Ar}$ plasma. The designed catalyst has the following advantages. First, the similar 2D geometries of $\mathrm{MoS}_{2}$ and defective graphene thin sheets enable the stabilization of S-vacancies on the stable 2D-2D interface. Second, the 2D natures of $\mathrm{MoS}_{2}$ and graphene result in growth of thin $\mathrm{MoS}_{2}$, and excellent electric contact between $\mathrm{MoS}_{2}$ and the conductive support. Third, the open structure and excellent wettability of the catalyst system facilitates the proton transport and access to the active sites. All of these features lead to superior HER activity, i.e., low overpotential, small Tafel slope, as well as outstanding long-term durability over $500 \mathrm{~h}$. Our strategy for stabilizing S-vacancies could be applicable to other catalysts beyond HER such as carbon dioxide reduction reaction. As all the components and processes are scalable, the demonstrated catalyst holds great promise as a scalable HER catalyst for PEM electrolyzers. 


\section{Declaration of Competing Interest}

The authors declare no competing financial interest.

\section{Acknowledgements}

The work was supported by Shell (China) Limited (award number PT78956). H.L. would like to thank Nanyang Technological University under NAP award (M408050000) and Tier 1 RG101/18 (2018-T1-001-051) for financial support. M.T.T. acknowledges support from the U.S. Department of Energy, Chemical Sciences, Geosciences, and Biosciences (CSGB) Division of the Office of Basic Energy Sciences, via Grant DE-AC02-76SF00515 to the SUNCAT Center for Interface Science and Catalysis at Stanford University. K.C. also acknowledges VILLUM FONDEN for a grant 9455.

\section{Appendix A. Supporting materials}

Supplementary data to this article can be found online at $\operatorname{xxxxx}$ 


\section{References}

[1] M. Carmo, D.L. Fritz, J. Mergel, D. Stolten, A comprehensive review on PEM water electrolysis, Int. J. Hydrogen Energy, 38 (2013) 4901-4934.

[2] M.G. Walter, E.L. Warren, J.R. McKone, S.W. Boettcher, Q. Mi, E.A. Santori, N.S. Lewis, Solar water splitting cells, Chem. Rev., 110 (2010) 6446-6473.

[3] S. Geng, W. Yang, Y.S. Yu, Building $\mathrm{MoS}_{2} / \mathrm{S}$-doped $\mathrm{g}_{-} \mathrm{C}_{3} \mathrm{~N}_{4}$ layered heterojunction electrocatalysts for efficient hydrogen evolution reaction, J. Catal., 375 (2019) 441-447.

[4] S. Sharma, S.K. Ghoshal, Hydrogen the future transportation fuel: from production to applications, Renewable Sustainable Energy Rev., 43 (2015) 1151-1158.

[5] D.M. Santos, C.A. Sequeira, J.L. Figueiredo, Hydrogen production by alkaline water electrolysis, Quim. Nova, 36 (2013) 1176-1193.

[6] M. Janjua, R. Le Roy, Electrocatalyst performance in industrial water electrolysers, Int. J. Hydrogen Energy, 10 (1985) 11-19.

[7] A. Ursua, L.M. Gandia, P. Sanchis, Hydrogen production from water electrolysis: current status and future trends, Proc. IEEE, 100 (2012) 410-426.

[8] P.C. Vesborg, T.F. Jaramillo, Addressing the terawatt challenge: scalability in the supply of chemical elements for renewable energy, RSC Adv., 2 (2012) 7933-7947.

[9] B. Hinnemann, P.G. Moses, J. Bonde, K.P. Jørgensen, J.H. Nielsen, S. Horch, I. Chorkendorff, J.K. Nørskov, Biomimetic hydrogen evolution: $\mathrm{MoS}_{2}$ nanoparticles as catalyst for hydrogen evolution, J. Am. Chem. Soc., 127 (2005) 5308-5309.

[10] T.F. Jaramillo, K.P. Jørgensen, J. Bonde, J.H. Nielsen, S. Horch, I. Chorkendorff, Identification of active edge sites for electrochemical $\mathrm{H}_{2}$ evolution from $\mathrm{MoS}_{2}$ nanocatalysts, Science, 317 (2007) 100-102.

[11] M.A. Lukowski, A.S. Daniel, F. Meng, A. Forticaux, L. Li, S. Jin, Enhanced hydrogen evolution catalysis from chemically exfoliated metallic $\mathrm{MoS}_{2}$ nanosheets, J. Am. Chem. Soc., 135 (2013) 10274-10277.

[12] C. Tsai, H. Li, S. Park, J. Park, H.S. Han, J.K. Nørskov, X. Zheng, F. Abild-Pedersen, Electrochemical generation of sulfur vacancies in the basal plane of $\mathrm{MoS}_{2}$ for hydrogen evolution, Nat. Commun., 8 (2017) 15113.

[13] Y. Xu, L. Wang, X. Liu, S. Zhang, C. Liu, D. Yan, Y. Zeng, Y. Pei, Y. Liu, S. Luo, Monolayer $\mathrm{MoS}_{2}$ with $\mathrm{S}$ vacancies from interlayer spacing expanded counterparts for highly efficient electrochemical hydrogen production, J. Mater. Chem. A, 4 (2016) 16524-16530.

[14] S. Yang, Y. Wang, H. Zhang, Y. Zhang, L. Liu, L. Fang, X. Yang, X. Gu, Y. Wang, Unique three-dimensional $\mathrm{Mo}_{2} \mathrm{C} @ \mathrm{MoS}_{2}$ heterojunction nanostructure with $\mathrm{S}$ vacancies as outstanding all-pH range electrocatalyst for hydrogen evolution, J. Catal., 371 (2019) 20-26.

[15] L. Lin, N. Miao, Y. Wen, S. Zhang, P. Ghosez, Z. Sun, D.A. Allwood, Sulfur-depleted monolayered molybdenum disulfide nanocrystals for superelectrochemical hydrogen evolution reaction, ACS Nano, 10 (2016) 8929-8937.

[16] H. Li, C. Tsai, A.L. Koh, L. Cai, A.W. Contryman, A.H. Fragapane, J. Zhao, H.S. Han, H.C. Manoharan, F. Abild-Pedersen, Activating and optimizing $\mathrm{MoS}_{2}$ basal planes for hydrogen evolution through the formation of strained sulphur vacancies, Nat. Mater., 15 (2016) 48.

[17] C.-C. Cheng, A.-Y. Lu, C.-C. Tseng, X. Yang, M.N. Hedhili, M.-C. Chen, K.-H. Wei, L.-J. Li, Activating basal-plane catalytic activity of two-dimensional $\mathrm{MoS}_{2}$ monolayer with remote hydrogen plasma, Nano Energy, 30 (2016) 846-852.

[18] Y. Bao, M. Yang, S.J.R. Tan, Y.P. Liu, H. Xu, W. Liu, C.T. Nai, Y.P. Feng, J. Lu, K.P. Loh, Substoichiometric molybdenum sulfide phases with catalytically active basal planes, J. Am. Chem. Soc., 138 (2016) 14121-14128.

[19] Y. Li, H. Wang, L. Xie, Y. Liang, G. Hong, H. Dai, $\mathrm{MoS}_{2}$ nanoparticles grown on 
graphene: an advanced catalyst for the hydrogen evolution reaction, J. Am. Chem. Soc., 133 (2011) 7296-7299.

[20] H. Li, M. Du, M.J. Mleczko, A.L. Koh, Y. Nishi, E. Pop, A.J. Bard, X. Zheng, Kinetic study of hydrogen evolution reaction over strained $\mathrm{MoS}_{2}$ with sulfur vacancies using scanning electrochemical microscopy, J. Am. Chem. Soc., 138 (2016) 5123-5129.

[21] H. Li, A.W. Contryman, X. Qian, S.M. Ardakani, Y. Gong, X. Wang, J.M. Weisse, C.H. Lee, J. Zhao, P.M. Ajayan, Optoelectronic crystal of artificial atoms in strain-textured molybdenum disulphide, Nat. Commun., 6 (2015) 7381.

[22] P. Giannozzi, S. Baroni, N. Bonini, M. Calandra, R. Car, C. Cavazzoni, D. Ceresoli, G.L. Chiarotti, M. Cococcioni, I. Dabo, QUANTUM ESPRESSO: a modular and open-source software project for quantum simulations of materials, J. Phys.: Condens. Matter, 21 (2009) 395502.

[23] A.H. Larsen, J.J. Mortensen, J. Blomqvist, I.E. Castelli, R. Christensen, M. Dułak, J. Friis, M.N. Groves, B. Hammer, C. Hargus, The atomic simulation environment-a Python library for working with atoms, J. Phys.: Condens. Matter, 29 (2017) 273002.

[24] J. Wellendorff, K.T. Lundgaard, A. Møgelhøj, V. Petzold, D.D. Landis, J.K. Nørskov, T. Bligaard, K.W. Jacobsen, Density functionals for surface science: Exchange-correlation model development with Bayesian error estimation, Phys. Rev. B, 85 (2012) 235149.

[25] C. Tsai, F. Abild-Pedersen, J.K. Nørskov, Tuning the MoS2 edge-site activity for hydrogen evolution via support interactions, Nano Lett., 14 (2014) 1381-1387.

[26] K. Chan, C. Tsai, H.A. Hansen, J.K. Nørskov, Molybdenum sulfides and selenides as possible electrocatalysts for $\mathrm{CO}_{2}$ reduction, ChemCatChem, 6 (2014) 1899-1905.

[27] N. Lopez, J.K. Nørskov, T. Janssens, A. Carlsson, A. Puig-Molina, B. Clausen, J.-D. Grunwaldt, The adhesion and shape of nanosized Au particles in a $\mathrm{Au} / \mathrm{TiO}_{2}$ catalyst, J. Catal., 225 (2004) 86-94.

[28] F. Studt, F. Abild- Pedersen, T. Bligaard, R.Z. Sørensen, C.H. Christensen, J.K. Nørskov, On the role of surface modifications of palladium catalysts in the selective hydrogenation of acetylene, Angew. Chem., Int. Ed., 47 (2008) 9299-9302.

[29] T.M.H. Dabros, A. Gaur, D.G. Pintos, P. Sprenger, M. Høj, T.W. Hansen, F. Studt, J. Gabrielsen, J.-D. Grunwaldt, A.D. Jensen, Influence of $\mathrm{H}_{2} \mathrm{O}$ and $\mathrm{H}_{2} \mathrm{~S}$ on the composition, activity, and stability of sulfided $\mathrm{Mo}$, CoMo, and $\mathrm{NiMo}$ supported on $\mathrm{MgAl}_{2} \mathrm{O}_{4}$ for hydrodeoxygenation of ethylene glycol, Appl. Catal., A, 551 (2018) 106-121.

[30] A. Gaur, T.M.H. Dabros, M. Høj, A. Boubnov, T. Pruessmann, J. Jelic, F. Studt, A.D. Jensen, J.-D. Grunwaldt, Probing the active sites of $\mathrm{MoS}_{2}$ based hydrotreating catalysts using modulation excitation spectroscopy, ACS Catal., (2019) 2568-2579.

[31] A.N. Enyashin, M. Bar-Sadan, L. Houben, G. Seifert, Line defects in molybdenum disulfide layers, J. Phys. Chem. C, 117 (2013) 10842-10848.

[32] W. Zhou, X. Zou, S. Najmaei, Z. Liu, Y. Shi, J. Kong, J. Lou, P.M. Ajayan, B.I. Yakobson, J.-C. Idrobo, Intrinsic structural defects in monolayer molybdenum disulfide, Nano Lett., 13 (2013) 2615-2622.

[33] D. Le, T.B. Rawal, T.S. Rahman, Single-layer $\mathrm{MoS}_{2}$ with sulfur vacancies: structure and catalytic application, J. Phys. Chem. C, 118 (2014) 5346-5351.

[34] S. Casolo, R. Martinazzo, G.F. Tantardini, Band engineering in graphene with superlattices of substitutional defects, J. Phys. Chem. C, 115 (2011) 3250-3256.

[35] Y. Wang, B. Chen, D.H. Seo, Z.J. Han, J.I. Wong, K.K. Ostrikov, H. Zhang, H.Y. Yang, $\mathrm{MoS}_{2}$-coated vertical graphene nanosheet for high-performance rechargeable lithium-ion batteries and hydrogen production, NPG Asia Mater., 8 (2016) e268.

[36] P.K. Chow, E. Singh, B.C. Viana, J. Gao, J. Luo, J. Li, Z. Lin, A.L. Elias, Y. Shi, Z. Wang, Wetting of mono and few-layered $\mathrm{WS}_{2}$ and $\mathrm{MoS}_{2}$ films supported on $\mathrm{Si} / \mathrm{SiO}_{2}$ substrates, ACS Nano, 9 (2015) 3023-3031. 
[37] G.R. Bhimanapati, T. Hankins, Y. Lei, R.A. Vilá, I. Fuller, M. Terrones, J.A. Robinson, Growth and tunable surface wettability of vertical $\mathrm{MoS}_{2}$ layers for improved hydrogen evolution reactions, ACS Appl. Mater. Interfaces, 8 (2016) 22190-22195.

[38] H. Huang, J. Huang, W. Liu, Y. Fang, Y. Liu, Ultradispersed and single-layered $\mathrm{MoS}_{2}$ nanoflakes strongly coupled with graphene: an optimized structure with high kinetics for the hydrogen evolution reaction, ACS Appl. Mater. Interfaces, 9 (2017) 39380-39390.

[39] A.B. Laursen, S. Kegnæs, S. Dahl, I. Chorkendorff, Molybdenum sulfides-efficient and viable materials for electro-and photoelectrocatalytic hydrogen evolution, Energy Environ. Sci., 5 (2012) 5577-5591.

[40] J.D. Benck, T.R. Hellstern, J. Kibsgaard, P. Chakthranont, T.F. Jaramillo, Catalyzing the hydrogen evolution reaction (HER) with molybdenum sulfide nanomaterials, ACS Catal., 4 (2014) 3957-3971.

[41] K.-C. Pham, Y.-H. Chang, D.S. McPhail, C. Mattevi, A.T. Wee, D.H. Chua, Amorphous molybdenum sulfide on graphene-carbon nanotube hybrids as highly active hydrogen evolution reaction catalysts, ACS Appl. Mater. Interfaces, 8 (2016) 5961-5971.

[42] Z. Liu, L. Zhao, Y. Liu, Z. Gao, S. Yuan, X. Li, N. Li, S. Miao, Vertical nanosheet array of $1 \mathrm{~T}$ phase $\mathrm{MoS}_{2}$ for efficient and stable hydrogen evolution, Appl. Catal., B, (2019) 296-320.

[43] T. Wu, G. Wang, Y. Zhang, S. Kang, H. Zhang, Electrochemical deposition of Pt on carbon fiber cloth utilizing Pt mesh counter electrode during hydrogen evolution reaction for electrocatalytic hydrogenation reduction of p-nitrophenol, New Journal of Chemistry, 41 (2017) 7012-7019.

[44] L. Zhang, L. Han, H. Liu, X. Liu, J. Luo, Potential- cycling synthesis of single platinum atoms for efficient hydrogen evolution in neutral media, Angew. Chem., Int. Ed., 56 (2017) 13694-13698.

[45] Y. Yan, X. Ge, Z. Liu, J.-Y. Wang, J.-M. Lee, X. Wang, Facile synthesis of low crystalline $\mathrm{MoS}_{2}$ nanosheet-coated CNTs for enhanced hydrogen evolution reaction, Nanoscale, 5 (2013) 7768-7771.

[46] G. Liu, A.W. Robertson, M.M.-J. Li, W.C. Kuo, M.T. Darby, M.H. Muhieddine, Y.-C. Lin, K. Suenaga, M. Stamatakis, J.H. Warner, $\mathrm{MoS}_{2}$ monolayer catalyst doped with isolated Co atoms for the hydrodeoxygenation reaction, Nat. Chem., 9 (2017) 810.

[47] Z. Luo, Y. Ouyang, H. Zhang, M. Xiao, J. Ge, Z. Jiang, J. Wang, D. Tang, X. Cao, C. Liu, Chemically activating $\mathrm{MoS}_{2}$ via spontaneous atomic palladium interfacial doping towards efficient hydrogen evolution, Nat. Commun., 9 (2018) 2120.

[48] Y. Yan, B. Xia, X. Ge, Z. Liu, J.-Y. Wang, X. Wang, Ultrathin $\mathrm{MoS}_{2}$ nanoplates with rich active sites as highly efficient catalyst for hydrogen evolution, ACS Appl. Mater. Interfaces, 5 (2013) 12794-12798.

[49] X. Zheng, J. Xu, K. Yan, H. Wang, Z. Wang, S. Yang, Space-confined growth of $\mathrm{MoS}_{2}$ nanosheets within graphite: the layered hybrid of $\mathrm{MoS}_{2}$ and graphene as an active catalyst for hydrogen evolution reaction, Chem. Mater., 26 (2014) 2344-2353.

[50] J.D. Benck, Z. Chen, L.Y. Kuritzky, A.J. Forman, T.F. Jaramillo, Amorphous molybdenum sulfide catalysts for electrochemical hydrogen production: insights into the origin of their catalytic activity, ACS Catal., 2 (2012) 1916-1923. 
Fig. 1. Rational design of stable sulfur vacancies in $\mathrm{MoS}_{2}$. (a) Schematic of the atomic structure of $\mathrm{MoS}_{2}$ with sulfur vacancies stabilized on defective graphene. The blue and yellow big balls represent Mo and $\mathrm{S}$ atoms, respectively. The grey small balls represent $\mathrm{C}$ atoms. The dotted red and blue dotted circle indicates $\mathrm{S}$ and $\mathrm{C}$ vacancies, respectively. Left panel: side view (upper) and top view (lower) of $\mathrm{MoS}_{2}$-graphene stack with $\mathrm{MoS}_{2}$ on top. A linear configuration of $\mathrm{S}$ vacancies is highlighted in the dashed box. Right panel: side view (upper) and top view (lower) of graphene- $\mathrm{MoS}_{2}$ stack with graphene on top. An aromatic ring configuration of $\mathrm{C}$-vacancies is highlighted by the dashed lines (missing $\mathrm{C}-\mathrm{C}$ bonds).

Fig. 2. Impact of S-vacancies in $\mathrm{MoS}_{2}$ and $\mathrm{C}$-vacancies in graphene on free energy. Reaction free energy versus the reaction coordinate of HER for the line-shaped S-vacancies range of 0-12.50\% supported on graphene with (a) $0 \%$ (pristine), (b) $1.85 \%$, (c) $3.70 \%$, (d) $5.56 \%$ (1 carbon ring missing), (e) $11.11 \%$ (2 carbon rings missing), and (f) $50 \%$ (edge). Insets: atomic configuration of graphene with various $\mathrm{C}$-vacancy\%. The blue and yellow balls represent Mo and $\mathrm{S}$ atoms, respectively. The grey balls represent $\mathrm{C}$ atoms, and the blue balls represent the missing $\mathrm{C}$ atoms.

Fig. 3. Stability of sulfur vacancies on defective graphene. (a) The adhesion energy per carbon atom of pristine $\mathrm{MoS}_{2}$ on defective graphene with carbon vacancies\%. Inset: Atomic configuration of $\mathrm{MoS}_{2}$ on various defective graphene substrates with graphene layer on top. The blue and yellow balls represent Mo and $\mathrm{S}$ atoms, respectively. The grey balls represent $\mathrm{C}$ atoms. The blue balls indicate the missing carbon atoms. Pristine graphene, graphene with one hexagon of carbon vacancies, graphene with two hexagons of carbon vacancies, and graphene edge from left to right are inserted. (b) The adhesion energy per carbon atom of $\mathrm{MoS}_{2}$ with S-vacancies on pristine graphene. Inset: atomic configuration of $\mathrm{MoS}_{2}$ with various S-vacancies\% on graphene substrate with $\mathrm{MoS}_{2}$ layer on top. The red balls indicate the missing sulfur atoms arranged in line shape. $\mathrm{MoS}_{2}$ with $0.00 \%$ (pristine), 3.13\%, 6.25\%, $9.38 \%$, and $12.50 \%$ S-vacancies from left to right are inserted. (c) Colored contour plot of adhesion energy as a function of S-vacancy $\%$ and C-vacancy\%. Inset: the color bar represents the value of adhesion energy.

Fig. 4. Material characterizations of the catalyst. SEM images of (a) vertical graphene network (VGN), (b) $\mathrm{MoS}_{2} / \mathrm{VGN}$ hybrid, and (c) $\mathrm{MoS}_{2} / \mathrm{VGN}$ hybrid with sulfur vacancies by Ar exposure for $120 \mathrm{~s}\left(\mathrm{~V}_{120}-\mathrm{MoS}_{2} / \mathrm{VGN}\right)$. The insets are the contact angle measurement results with $147^{\circ}, 131^{\circ}$ and $25^{\circ}$ from (a) to (c). The scale bars in (a) to (c) are $500 \mathrm{~nm}$. (d) Raman spectra of V-MoS $2 / \mathrm{VGN}$ and $\mathrm{MoS}_{2} / \mathrm{VGN} @ \mathrm{CP}$ heterostructure. (e) HRTEM image of 
$\mathrm{MoS}_{2} / \mathrm{VGN}$. The scale bar is $10 \mathrm{~nm}$. (f) TEM image of $\mathrm{V}_{120}-\mathrm{MoS}_{2} / \mathrm{VGN}$. Inset: corresponding HRTEM image. The scale bar in (f) is $100 \mathrm{~nm}$, and that in the inset is $10 \mathrm{~nm}$. The interlayer distance of graphene $(\sim 3 \AA)$ and $\mathrm{V}-\mathrm{MoS}_{2}(\sim 6 \AA)$ are both labelled in (e) and (f).

Fig. 5. Electrochemical test and S-Vacancy characterization of the catalyst. (a) LSV curves and (b) Tafel plots of the $\mathrm{V}_{120}-\mathrm{MoS}_{2} / \mathrm{VGN} @ \mathrm{CP}, \mathrm{MoS}_{2} / \mathrm{VGN} @ \mathrm{CP}, \mathrm{MoS}_{2} @ \mathrm{CP}, \mathrm{VGN} @ \mathrm{CP}$, $\mathrm{CP}$ and Pt. The Tafel slope of each curve is labelled. (c) Stability test of the $\mathrm{V}_{120}-\mathrm{MoS}_{2} / \mathrm{VGN} @ \mathrm{CP}$ up to $500 \mathrm{~h}$, where the inset is SEM image after stability test. (d) Overpotential (at $10 \mathrm{~mA} \mathrm{~cm}^{-2}$ ) versus Ar plasma treatment duration and the red line is fitting curve. (e) EPR spectra of $\mathrm{V}_{\mathrm{t}}-\mathrm{MoS}_{2} / \mathrm{VGN} @ \mathrm{CP}$ when $\mathrm{t}$ is increased from 0 (i.e., $\mathrm{MoS}_{2} / \mathrm{VGN} @ \mathrm{CP}$ ) to 40, 80, 120, and 160 s. (f) XPS spectra of $\mathrm{S} 2 \mathrm{p}$ states of $\mathrm{V}_{\mathrm{t}}-\mathrm{MoS}_{2} / \mathrm{VGN} @ \mathrm{CP}$ sample when $\mathrm{t}$ increases from 0 to 80, 100, 120, 140, $160 \mathrm{~s}$. $\mathrm{V}_{120}-\mathrm{MoS}_{2} / \mathrm{VGN} @ \mathrm{CP}$ (i.e., $\mathrm{t}=120$ ) case is highlighted in red. 


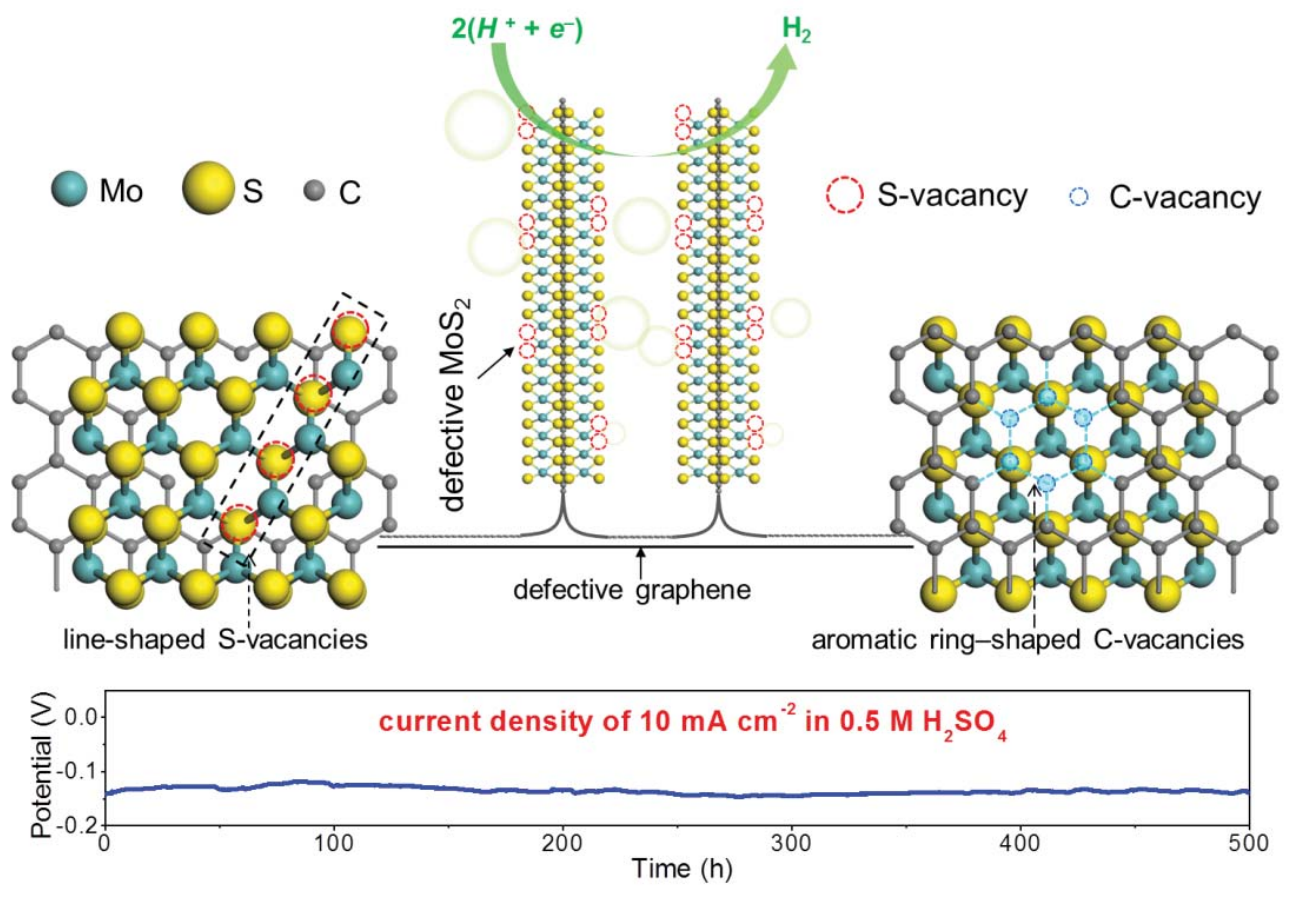


- A MoS 2 catalyst consists of sulfur vacancies is rationally designed for acidic HER.

- The $\mathrm{MoS}_{2}$ with line-shaped vacancies is stabilized on vertical graphene network.

- The designed HER catalyst shows stable operation over 500 hours at $10 \mathrm{~mA} \mathrm{~cm}$.

- DFT calculations show the mechanisms for stable HER reaction. 
Figure 1

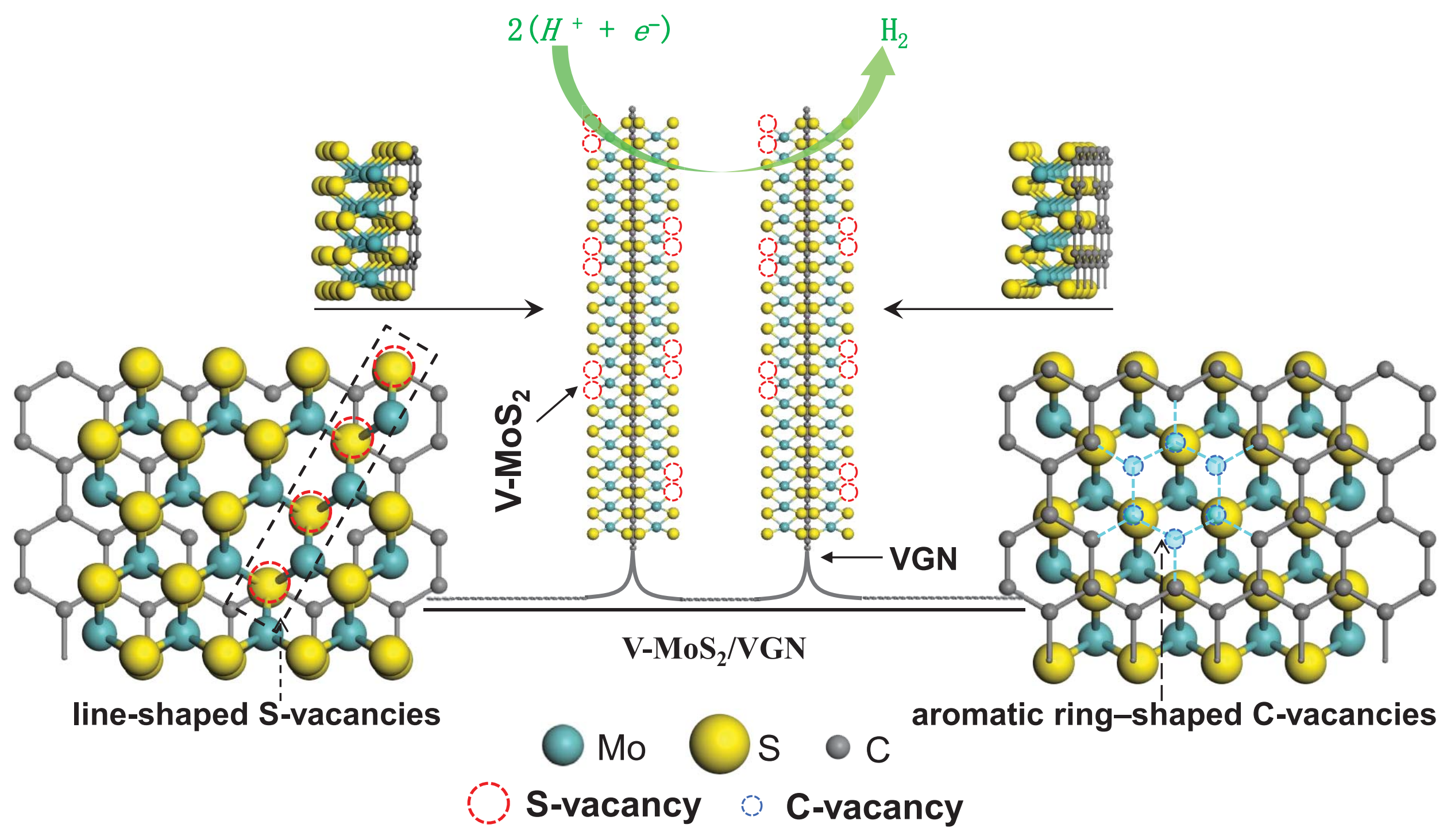


(iagre

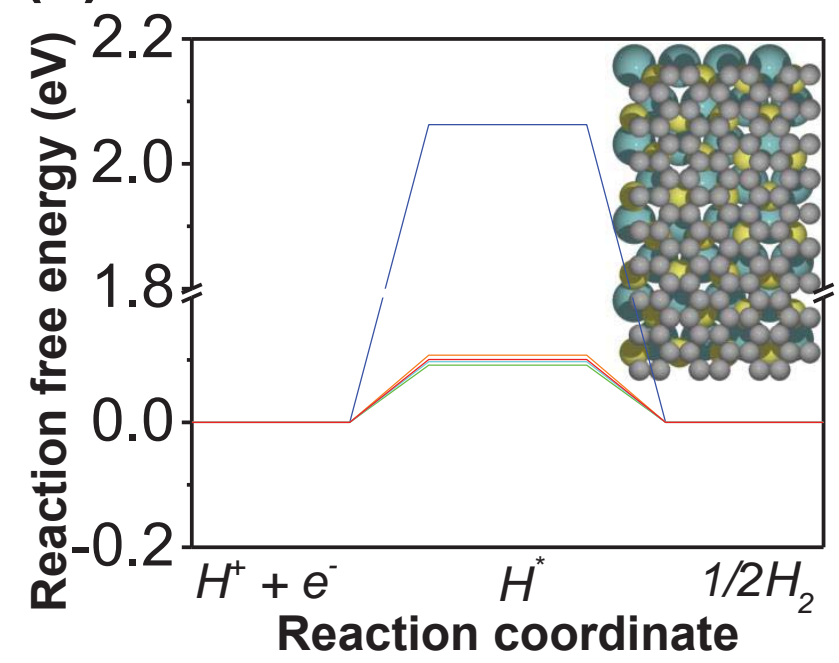

- 0\% S-vacancy
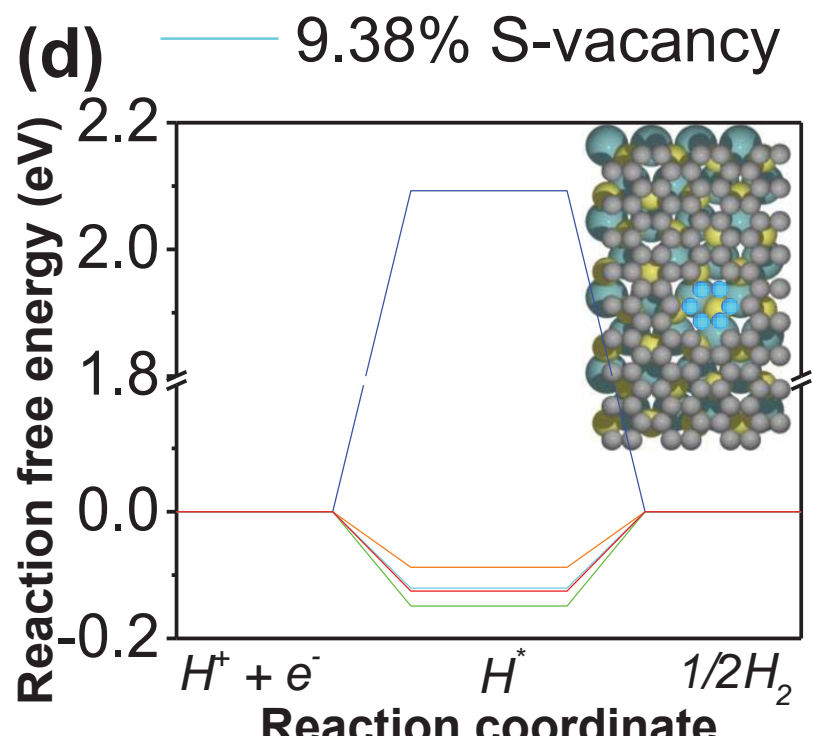

(b)

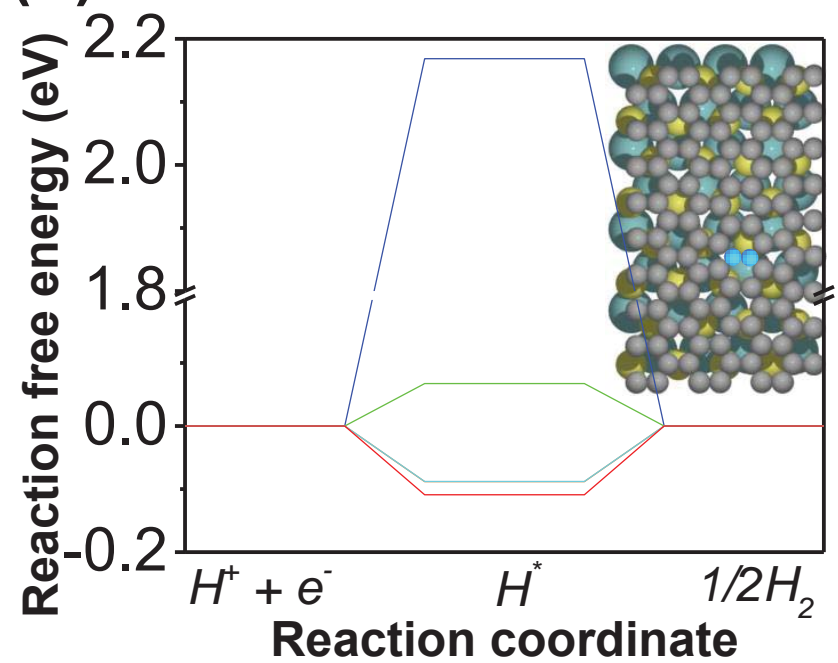

$3.13 \%$ S-vacancy

(e)

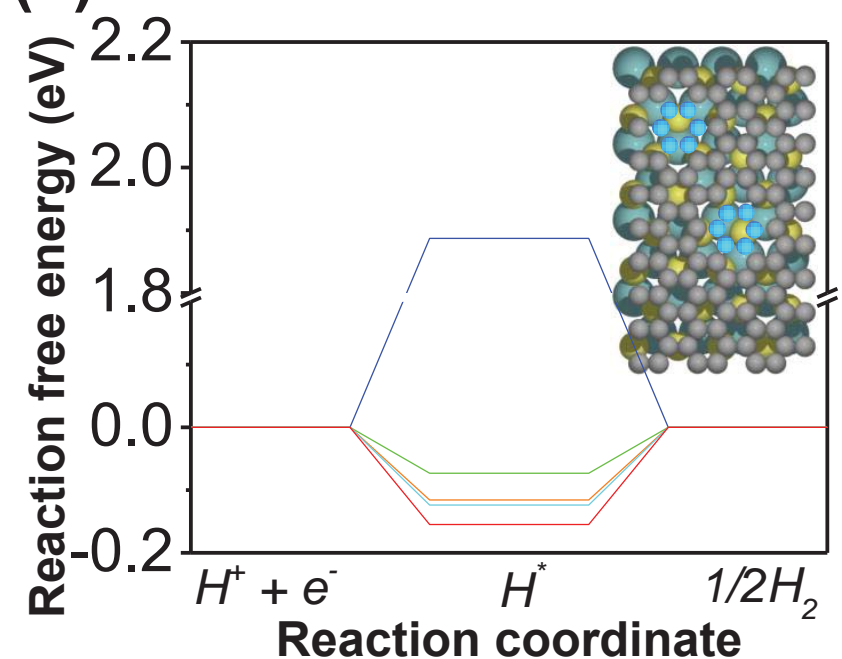

(c)

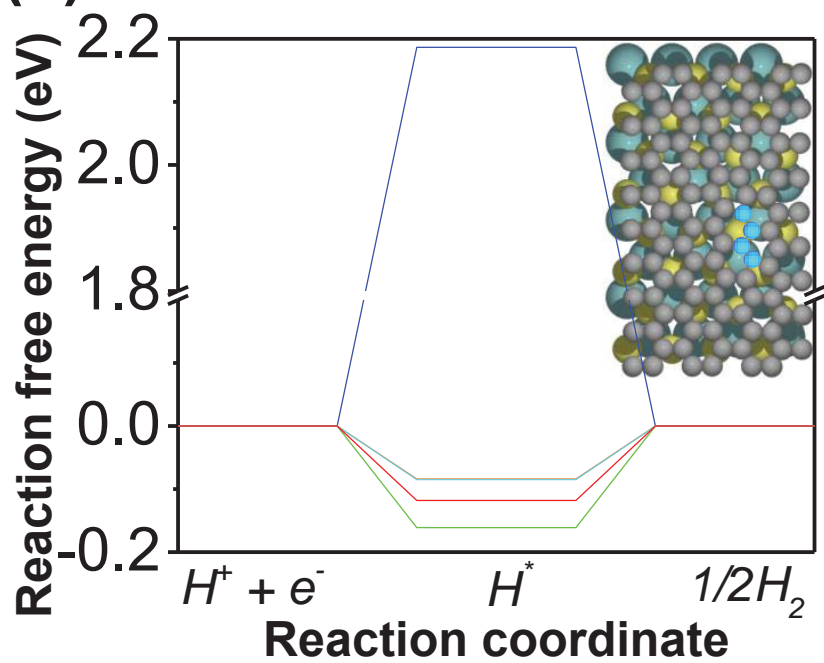

$6.25 \%$ S-vacancy

(f)

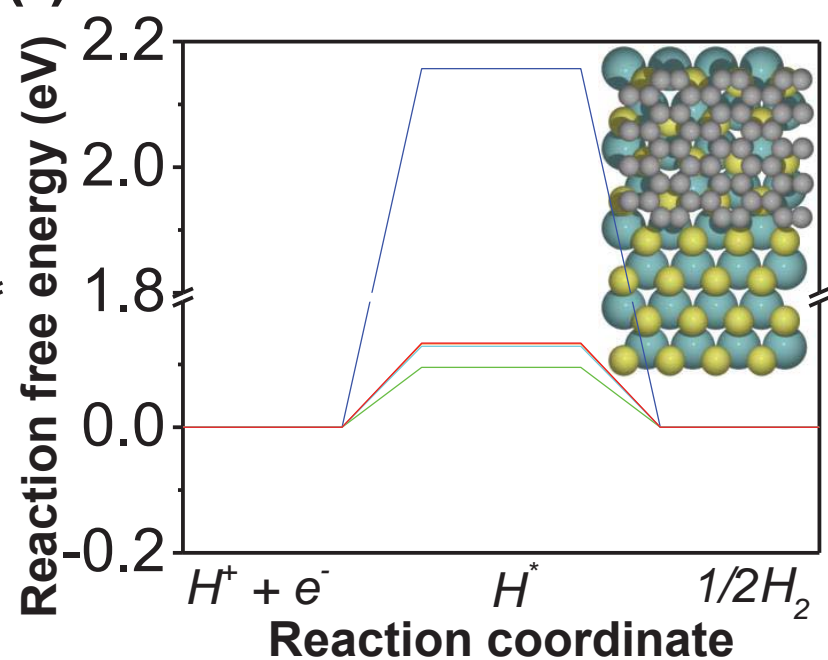


(a)

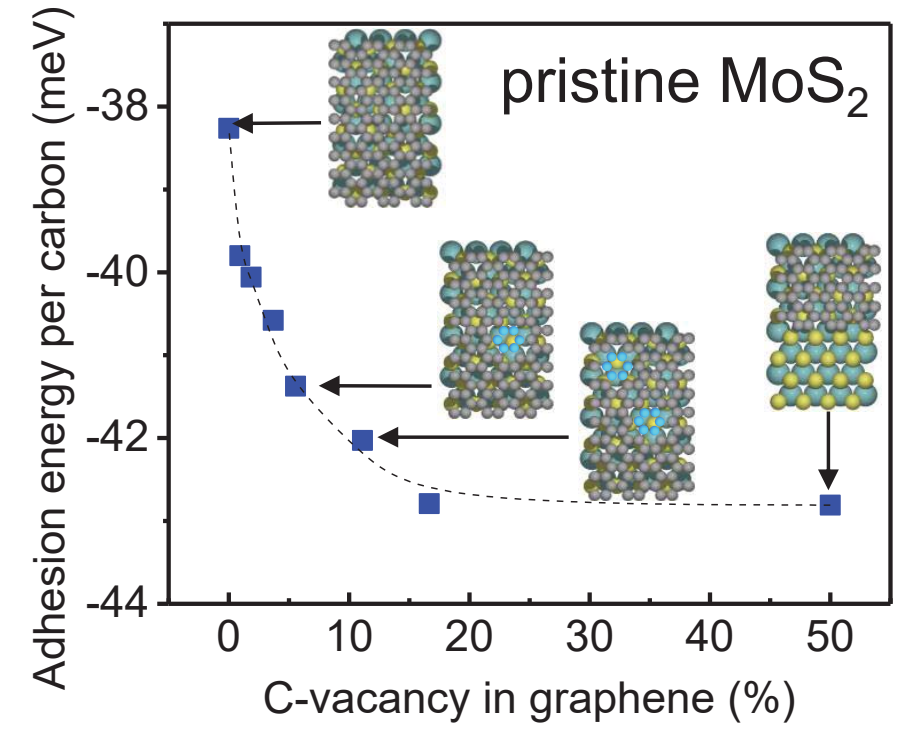

(c)

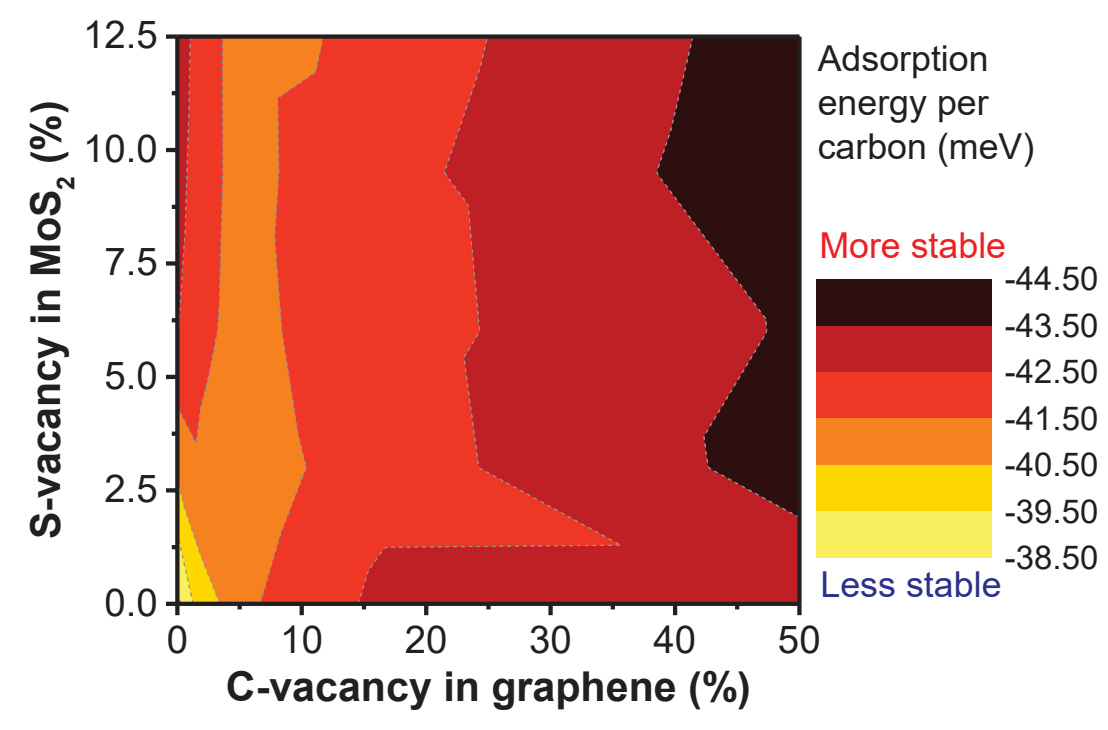

(b)

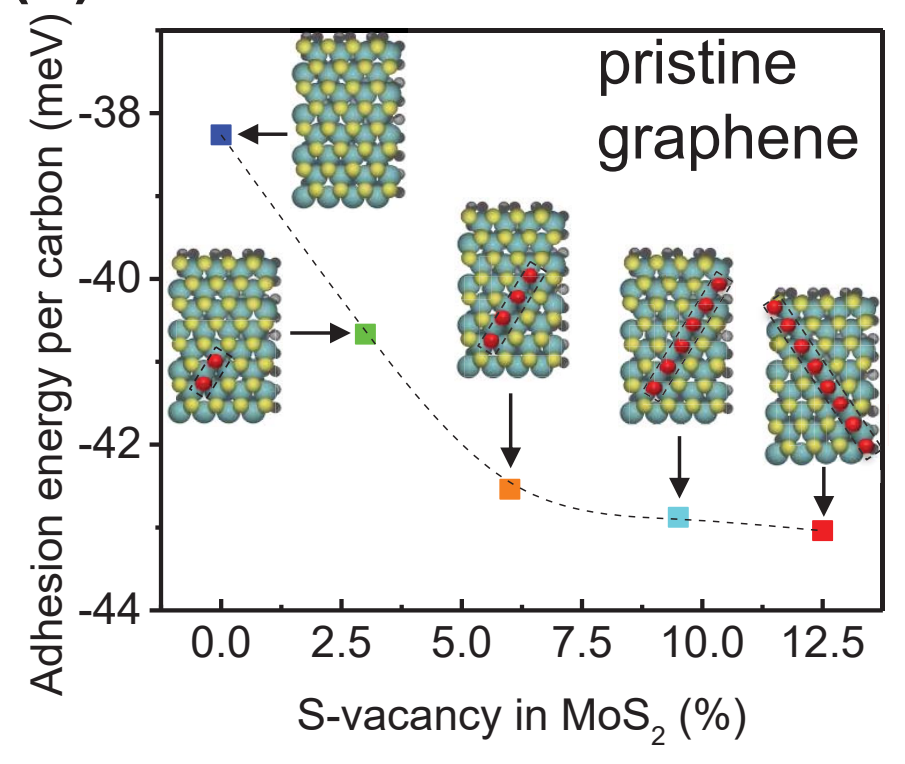


(a)

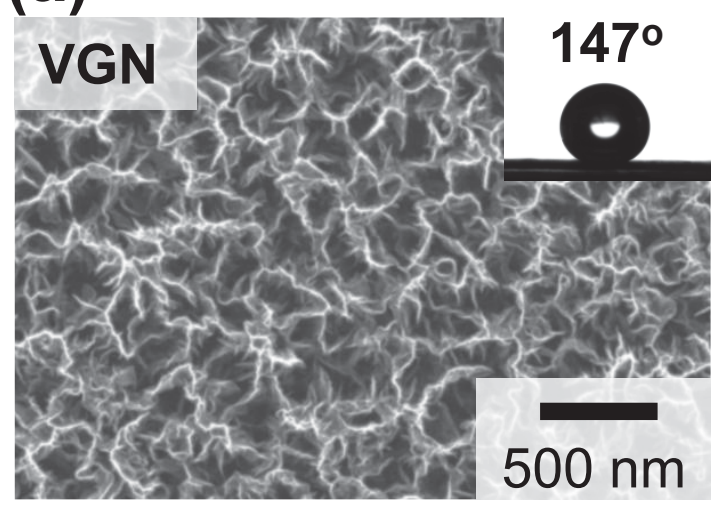

(d)

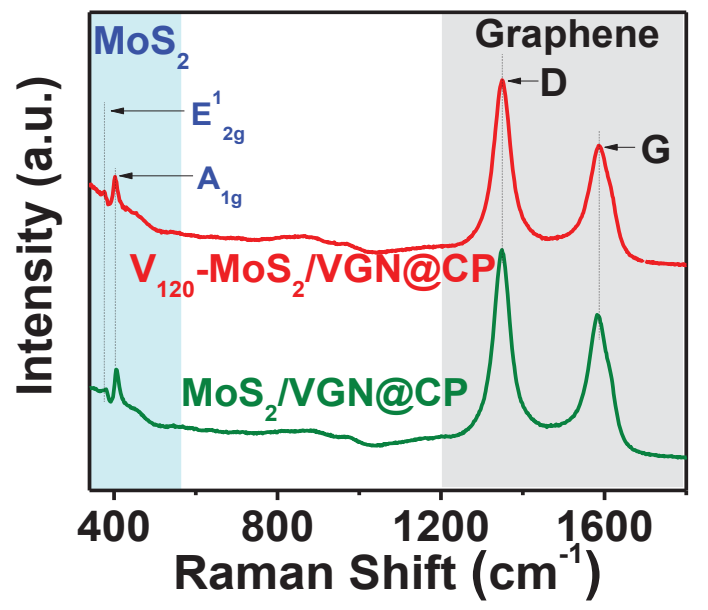

(b)

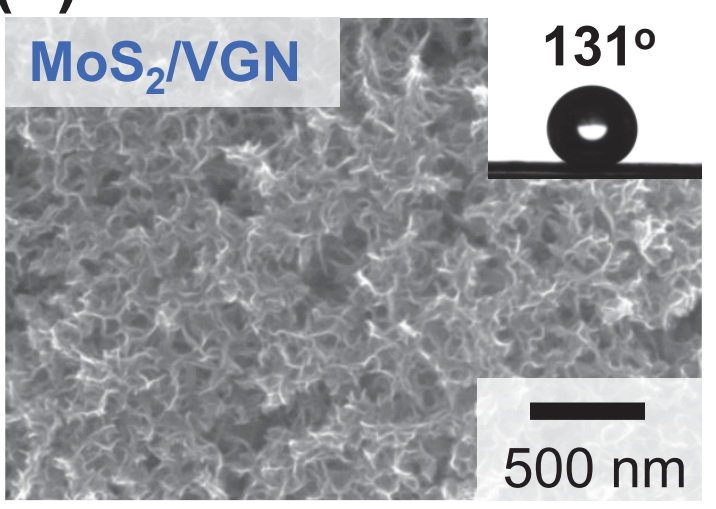

(e)

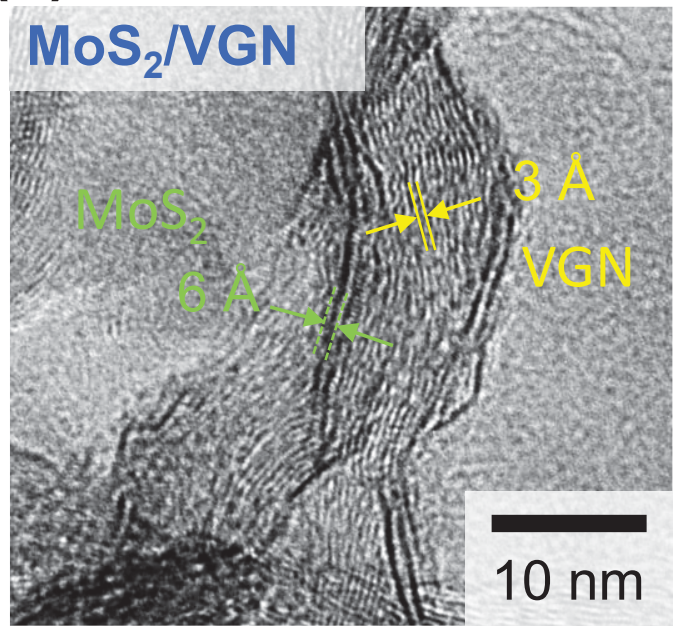

(c)

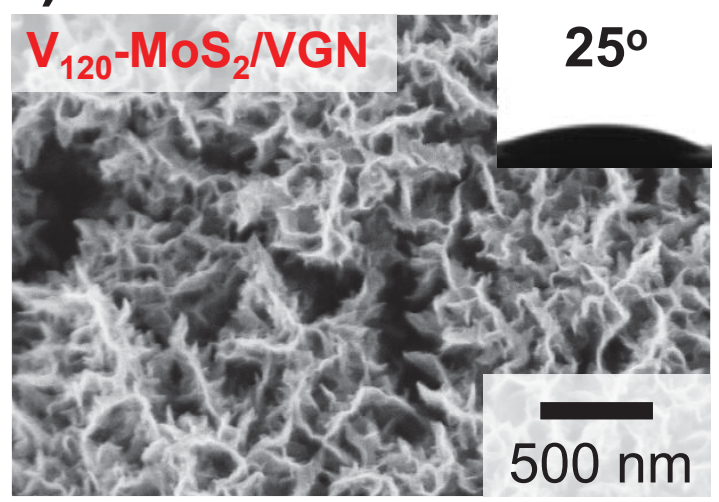

(f)

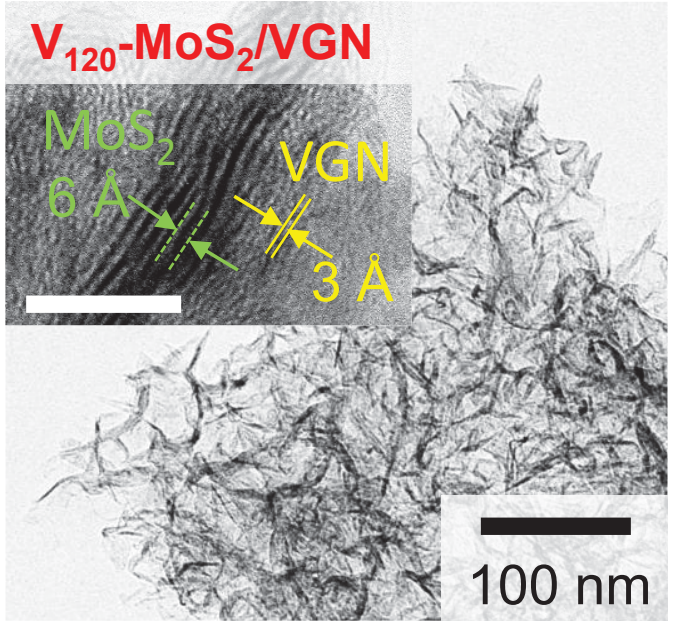


(a)

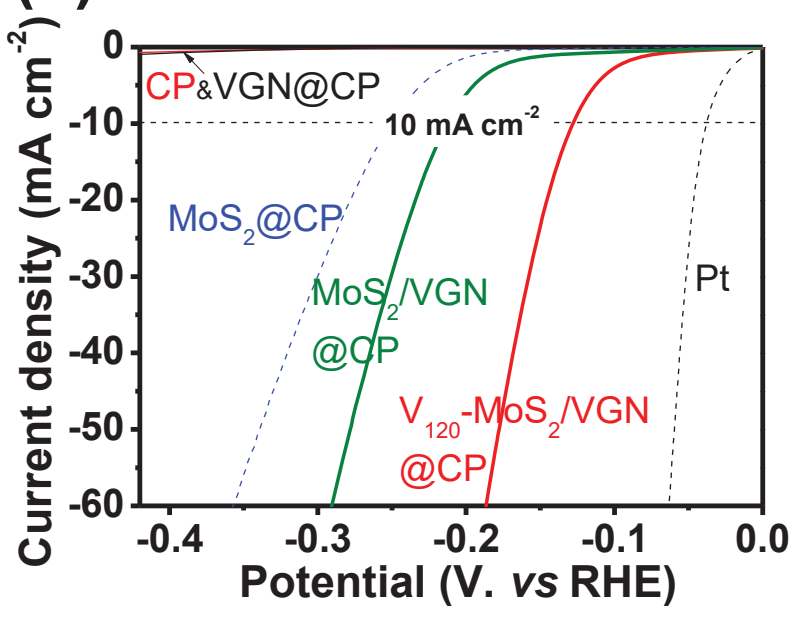

(d)

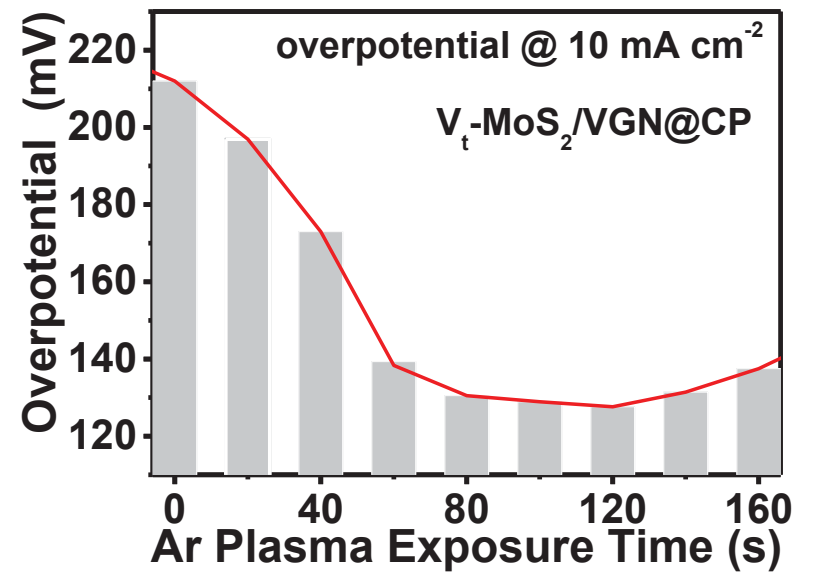

(b)

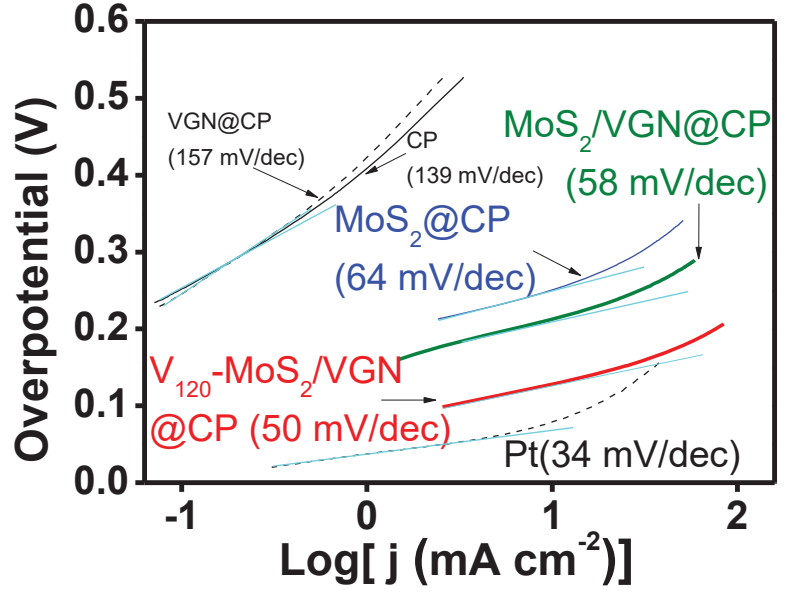

(e)

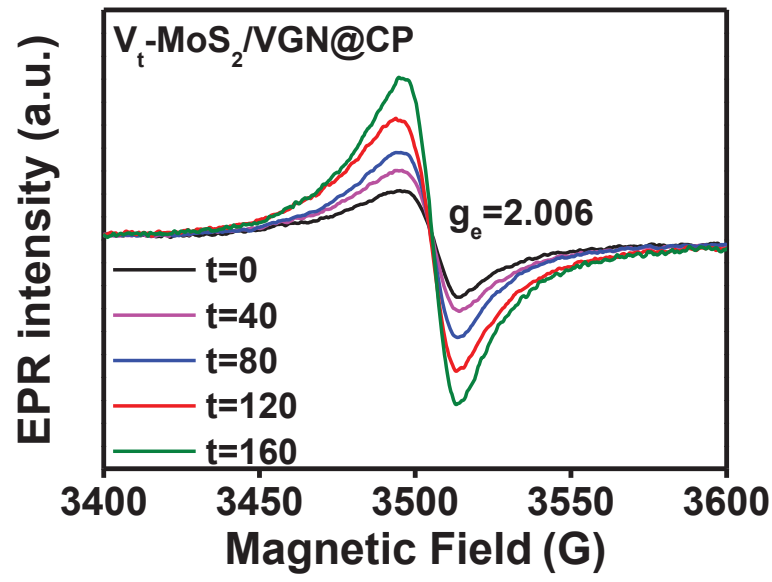

(c)

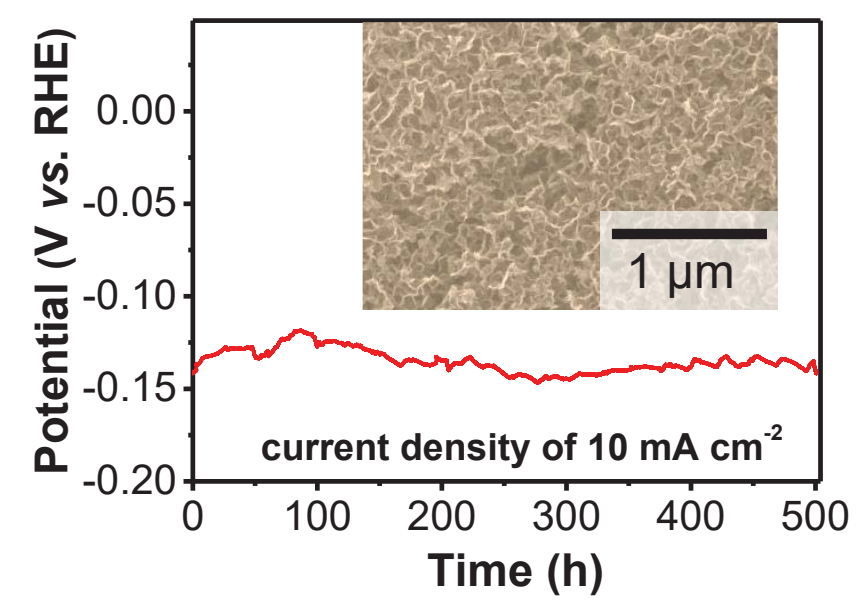

(f)

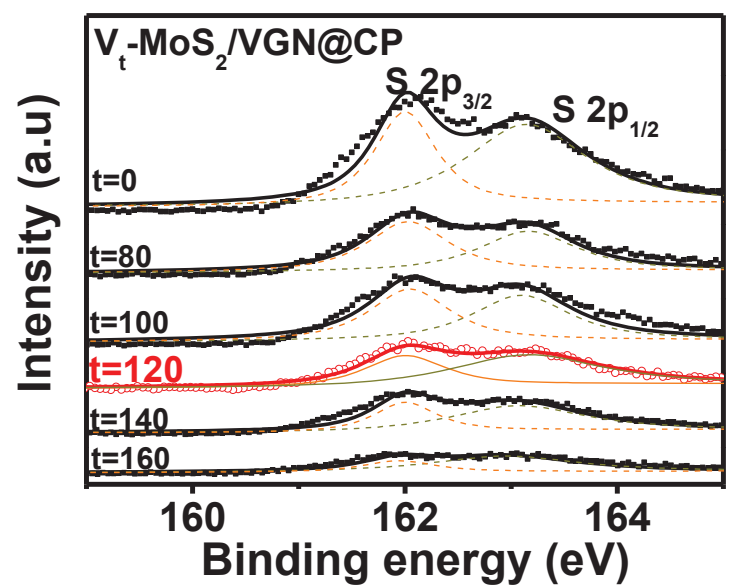

